\title{
HDAC4 degradation during senescence unleashes an epigenetic program driven by AP-1/p300 at selected enhancers and super-enhancers
}

Eros Di Giorgio ${ }^{1}$, Harikrishnareddy Paluvai ${ }^{1}$, Emiliano Dalla ${ }^{1}$, Liliana Ranzino ${ }^{1}$, Alessandra Renzini², Viviana Moresi ${ }^{2}$, Martina Minisini ${ }^{1}$, Raffaella Picco ${ }^{1}$ and Claudio Brancolini ${ }^{1 *}$ (D)

\section{* Correspondence: claudio}

brancolini@uniud.it

${ }^{1}$ Department of Medicine, Università

degli Studi di Udine, p.le Kolbe 4,

33100 Udine, Italy

Full list of author information is

available at the end of the article

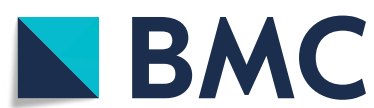

\section{Background}

Cellular senescence and aging are complex responses characterized by proliferative arrest and loss of regenerative potential [1]. The senescence state is distinguished by a deep epigenetic reprogramming that sculptures the chromatin to arrest the cell cycle, maintain cellular survival, and secrete apocrine and paracrine factors [2].

(c) The Author(s). 2021 Open Access This article is licensed under a Creative Commons Attribution 4.0 International License, which permits use, sharing, adaptation, distribution and reproduction in any medium or format, as long as you give appropriate credit to the original author(s) and the source, provide a link to the Creative Commons licence, and indicate if changes were made. The images or other third party material in this article are included in the article's Creative Commons licence, unless indicated otherwise in a credit line to the material. If material is not included in the article's Creative Commons licence and your intended use is not permitted by statutory regulation or exceeds the permitted use, you will need to obtain permission directly from the copyright holder. To view a copy of this licence, visit http://creativecommons.org/licenses/by/4.0/. The Creative Commons Public Domain Dedication waiver (http://creativecommons.org/publicdomain/zero/1.0/) applies to the data made available in this article, unless otherwise stated in a credit line to the data. 
Alterations in the H3K27ac/H3K27me3 ratios were identified as the driving forces of premature senescence and cancer [3-5]. The epigenetic control at distal regulatory elements is an important switch in the establishment of the senescent status [6, 7]. The histone acetyltransferase p300 promotes the formation of active enhancer elements that propel the senescence-specific gene expression program [5]. The transcription factor (TF) activator protein 1 (AP-1) is a key regulator of the transcriptional program required to launch the oncogene-induced senescence (OIS). This activity is supervised through the binding of senescence enhancers [8]. AP-1 can act as a pioneer onto the senescence enhancers to orchestrate the transcriptional program of senescent cells [8, 9].

Although some knowledges have been acquired during the last years on enhancers and super-enhancers activation in senescence, the mechanisms counteracting this activation are much less characterized. Enhancer regions are defined by characteristic histone modifications (e.g., H3K27ac and H3K4me1) [10]. The balanced action of acetyltransferases (HAT) such as p300/CBP, by interacting with the SWI/SNF complex [11] and HDACs of the Sin3, NuRD, CoREST, MiDAC, and NCOR complexes [12, 13], controls the acetylation status of H3K27. In vertebrates, class IIa HDACs are catalytically inactive epigenetic readers, quickly recruited on H3K27ac loci [14-17]. Here, they can monitor the acetylation status of H3K27 through the interaction with class I HDACs [18]. Class IIa HDACs supervise specific differentiation programs and different adaptive responses. Dysregulations of class IIa HDACs can contribute to cancer development and other diseases [18, 19]. In this manuscript, we have investigated in a comprehensive manner the regulation of class IIa HDACs during senescence and aging. Our results point to a key role of HDAC4 in counteracting replicative senescence and oncogene-induced senescence (OIS). HDAC4 by monitoring H3K27ac levels at selected enhancers and super-enhancers antagonizes the activation of the senescence gene expression program.

\section{Results}

HDAC4 expression is downregulated during different models of cellular senescence and with aging

The epigenetic reprogramming plays essential roles in establishing cellular senescence and aging. In this context, the contribution of class IIa HDACs has been suggested [20, 21], but not addressed in a comprehensive manner. It is known that important epigenetic modulators of senescence and aging are downregulated during senescence onset $[4,22,23]$. Hence, we initially evaluated class IIa HDACs expression levels in different models of senescence and aging. HDAC4 and HDAC9, and to a lesser extent HDAC7, are progressively downregulated in human IMR90 fibroblasts undergoing replicative senescence, with a timing similar to Lamin-B1 (Fig. 1a/b). As a model of aging, we compared the levels of class IIa HDACs in the dermis and in the liver of young (4 months) and old ( 25 months) female mice. In humans, female longevity tends to exceed male longevity; for this reason, we investigated Hdac4 levels specifically in female mice [24]. HDAC4 and HDAC9 levels decrease, both in aged dermis and liver, whereas HDAC5 decreases only in old dermis. As expected, aged tissues are characterized by high TP53 levels and Lamin-B1 downregulation (Fig. 1c). In agreement with our data, in mice representing a senescence-accelerated model of aging, the downregulation of HDAC4 was previously observed [25]. Therefore, telomere attrition in normal cells and physiological aging, similarly affect HDAC4 and HDAC9 protein levels. 


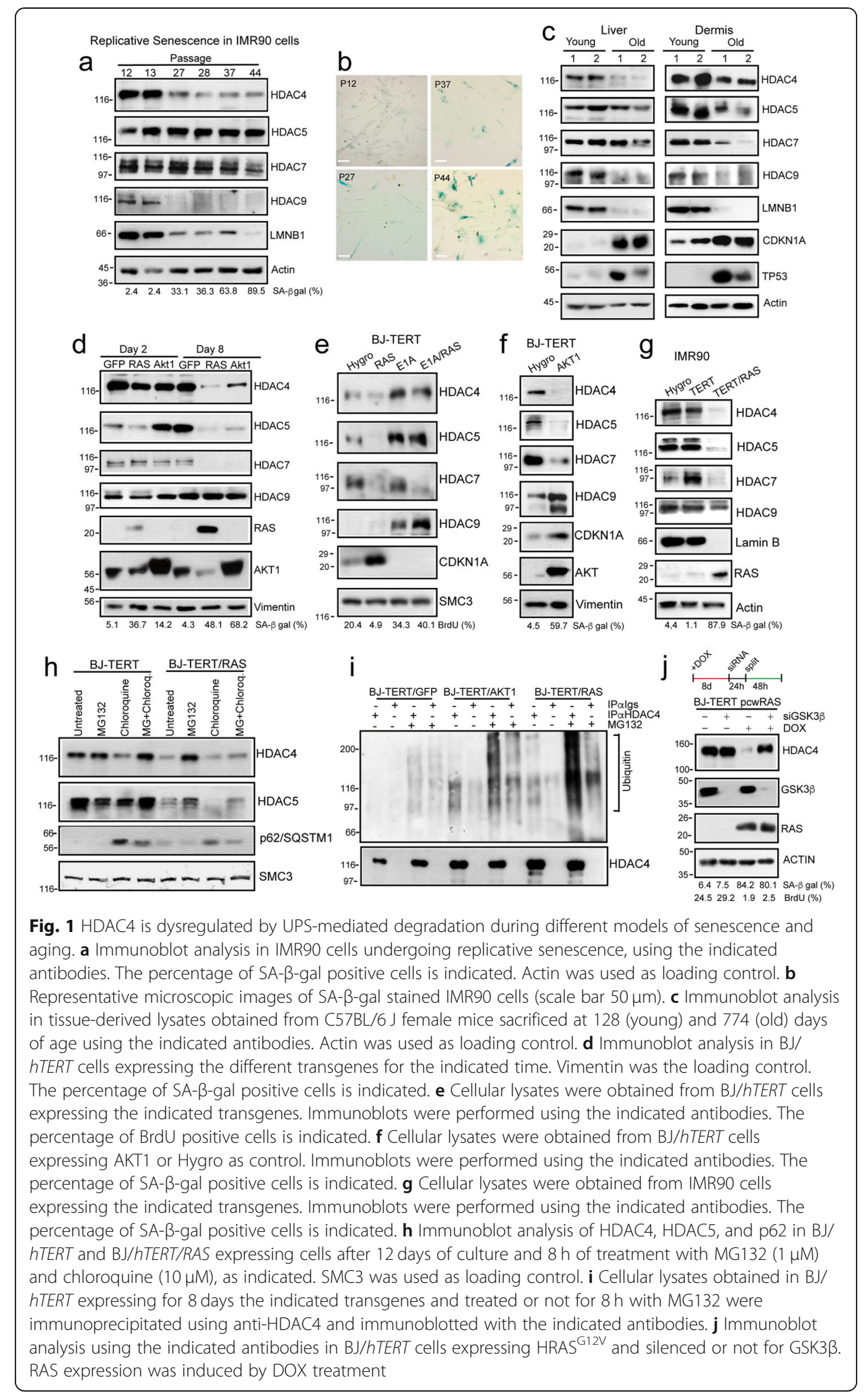

Next, we used additional models of cellular senescence to investigate the behavior of class IIa HDACs: oncogene-induced senescence (OIS) [26, 27], stress-induced senescence (SISP), following $\mathrm{H}_{2} \mathrm{O}_{2}$ treatment, and cytokine-induced senescence [28]. The 
induction of senescence was verified by classical markers (SA- $\beta$ gal staining positivity, BrdU negativity, CDKN1A upregulation and LMNB1 downregulation). The delivery in $\mathrm{BJ} / h T E R T$ cells of the strong oncogenes HRAS ${ }^{\mathrm{G} 12 \mathrm{~V}}$ (thereafter RAS) or myrAKT1 triggers OIS (Additional file 1: Fig. S1a). In these inducible models of OIS, the downregulation of HDAC4, HDAC5, and HDAC7 occurs after the initial proliferative phase, at day 8 from oncogene induction (Fig. 1d). Class IIa HDACs downregulation is coupled to the upregulation of CDKN1A, CDKN2A, and GADD45A transcription (Additional file 1: Fig. S1b).

Oncogenes of viral origin, like E1A and its $\triangle \mathrm{C}$-fragment (1-143), are able to overcome OIS, by targeting the p16/Rb pathways [29]. HDAC4/5/9, but not HDAC7, are upregulated by the expression of E1A $\triangle \mathrm{C}$ in BJ/hTERT cells (Fig. 1e). Moreover, the coexpression of E1A and RAS bypasses the senescent arrest and recovers the protein levels of HDAC4 and HDAC5, but not of HDAC7 (Fig. 1e). The same regulation of class IIa HDACs was confirmed in a second model of AKT1-induced OIS (Fig. 1f) and in IMR90 fibroblasts during RAS-induced OIS (Fig. 1g). Curiously, RAS triggers a reduction of HDAC9 levels in IMR90 cells compared to BJ cells. The higher percentage of SA- $\beta$-gal positive cells suggests that IMR90/TERT/RAS cells are in a more advanced state of senescence compared to BJ/TERT/RAS cells. HDAC4 and HDAC5 are decreased also during SISP and cytokine-induced senescence (Additional file 1: Fig. S1c/ d). In summary, our screening identified HDAC4 as the class IIa HDAC member repressed in all tested models of senescence and in aging.

Senescence marginally affects class IIa HDACs mRNA levels. The exception was HDAC9, whose mRNA increases in response to oncogene activation (Additional file 1: Fig. S1e/f/g). Therefore, the downregulation of HDAC4 during OIS could be mediated by the ubiquitin-proteasome system (UPS) as demonstrated in other contexts [30]. During OIS, HDAC4 levels are restored by the proteasome inhibitor MG132 but not after autophagy inhibition (Fig. 1h). Curiously, autophagy blockage suppresses HDAC4 expression independently from OIS. Accordingly, HDAC4 is highly poly-ubiquitylated in senescent cells (Fig. 1i and Additional file 1: Fig. S1h). Previous studies have shown that the ubiquitin-dependent degradation of HDAC4 depends on GSK3 $\beta$ [30]. Similarly, in senescent cells, the treatment with $\mathrm{LiCl}$ (Additional file 1: Fig. S1i) and the silencing of GSK3 3 (Fig. 1j) are both capable of restoring HDAC4 levels.

Class IIa HDACs nuclear/cytoplasmic shuttling is intensively regulated by phosphorylation. We investigated the levels of serines $246 / 259$ phosphorylation, found respectively in HDAC4 and HDAC5, during RAS-induced senescence (RIS). These serines, that are binding sites for 14-3-3 proteins and involved in the nuclear export, are rapidly dephosphorylated upon RAS induction (Additional file 1: Fig. S1j). However, HDAC4 shows a pan/nuclear localization in BJ-TERT cells, and this localization is largely unperturbed after RAS activation (Additional file 1: Fig. S1k). In conclusion, dephosphorylation at serines $246 / 259$ is an early response to RAS induction, coupled to the proliferative boost.

The depletion of HDAC4 anticipates senescence while its re-expression delays RASinduced senescence (RIS)

We established different cellular models to investigate the role played by HDAC4 in regulating senescence onset and escape. Firstly, we conditionally knocked-out Hdac4 
(4-OHT dependent) in primary murine embryonic fibroblasts (MEFs). MEFs undergo senescence rapidly when grown at atmospheric oxygen, while the maintenance of hypoxic conditions preserves their proliferation [31]. The conditional $\mathrm{KO}$ of Hdac4 in MEFs under normoxia anticipates the progressive increase in Cdkn2a/p16 levels (Additional file 1: Fig. S2a). Accordingly, $\mathrm{Hdac}^{-/-}$MEFs arrest the proliferation earlier (Additional file 1: Fig. S2b/c) and anticipate the upregulation of genes related to senescence such as $C d k n 2 a, I l 1 b$, Ifnb, and irf7 (Additional file 1: Fig. S2d). As expected, when MEFs are grown in hypoxic conditions senescence is not observed (Additional file 1: Fig. S2e/f). However, prior to immortalization, $\mathrm{Hdac}^{{ }^{-/-}} \mathrm{MEFs}$ experience a proliferative crisis between 40 and 50 days of culture (Additional file 1: Fig. S2f).

Secondly, we tested the role played by HDAC4 in regulating OIS escape in human cells. The BJ/hTERT/Ras/E1A cells are a classical model of OIS escape [29]. CRISPRmediated HDAC4 KO (Fig. 2a and Additional file 1: Fig. S3a/b) in BJ/hTERT/Ras/E1A cells causes the appearance of SA- $\beta$-gal positive cells (Fig. $2 \mathrm{~b}$ ) and triggers the expression of senescence-related genes (Fig. 2c). The induction of senescence in these cells is unrelated to E1A levels (Fig. 2a).

To further characterize HDAC4 as an important element in counteracting OIS, we evaluated its ability to reverse OIS upon RAS induction. The effect of HDAC4 was compared to the silencing of pRB, TP53, or NFKB pathways, well-known regulators of the senescence response. They are involved in the control of the cell cycle (pRB), in sensing oncogenic lesions/DNA damage (TP53), and in SASP surveillance (NFKB) $[2$,

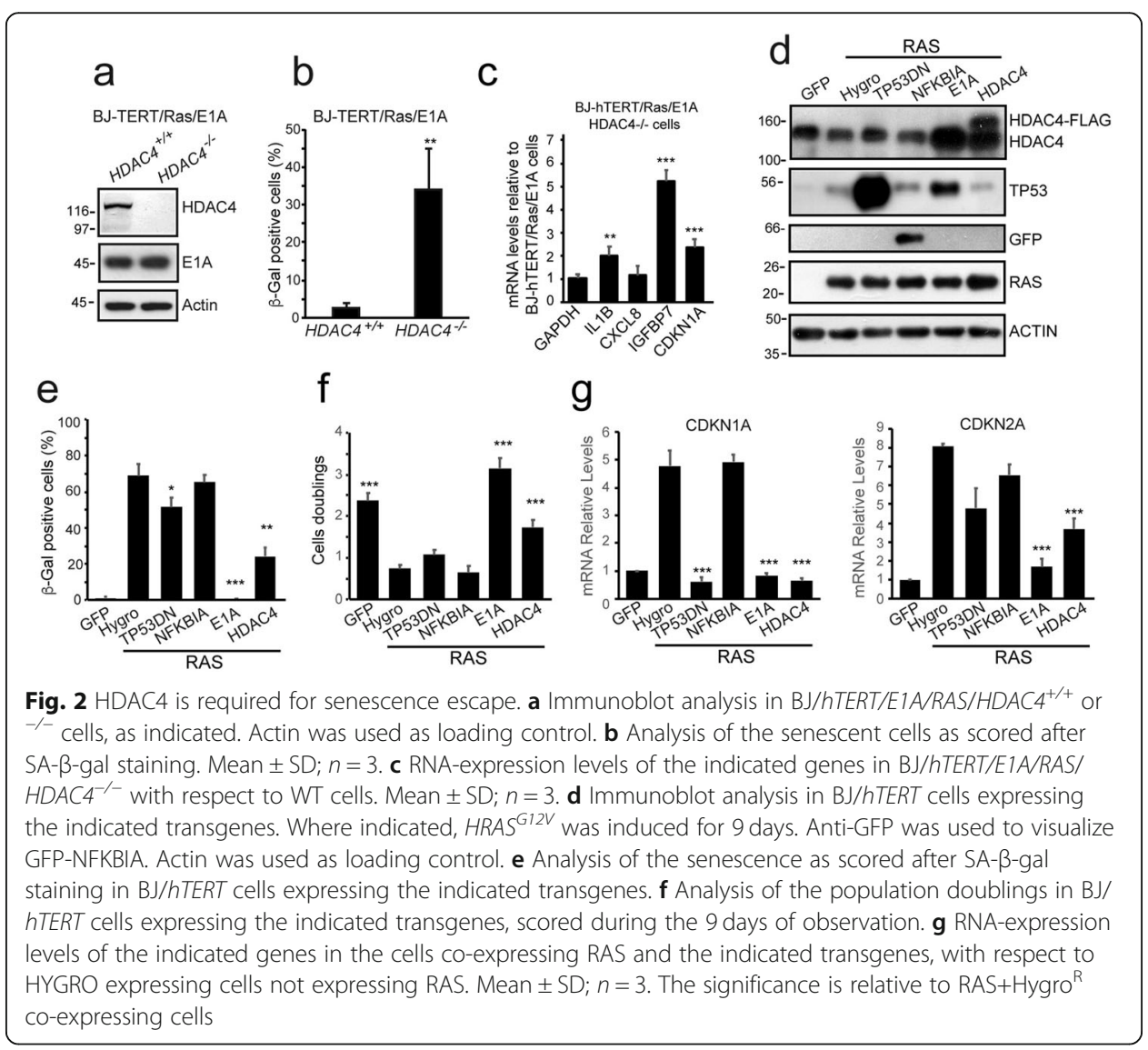


28]. E1A, the TP53DN dominant negative mutant (TP53 ${ }^{\mathrm{R} 175 \mathrm{H}}$ ) [32], the NFKB inhibitor IKB $\alpha$ S32A/S36A [33], referred hereinafter as NFKBIA, and HDAC4 were overexpressed in $\mathrm{BJ} / \mathrm{h} T E R T$ cells induced to express RAS for 9 days to reach OIS. Increasing the levels of HDAC4 in BJ/hTERT/RAS-ER cells decreases senescence onset (Fig. 2e) and sustains cell proliferation (Fig. 2f). HDAC4 also represses CDKN1A and CDKN2A expression (Fig. 2g). Only E1A shows a stronger OIS bypass activity compared to HDAC4.

\section{Depletion of HDAC4 triggers senescence in cancer cells}

Since class IIa HDACs are abundantly expressed in leiomyosarcomas (LMS) [15, 34], to confirm the role of HDAC4 in senescence escape, its expression was knocked-out also in low grade LMS cells SK-LMS-1 [35] (Fig. 3a and Additional file 1: Fig. S3a/b). In this additional model of OIS escape, HDAC4 depletion arrests proliferation (Fig. 3b) and induces SA- $\beta$-gal positivity (Fig. $3 \mathrm{c}$ and Additional file 1 : S3c). All KO clones fail to grow in semisolid medium (Additional file 1: Fig. S3d/e). These phenotypes are reproducible in the 4 KO clones generated with 2 different sgRNAs (sg1: 76, 1231; sg2: 205, 1254). Removal of HDAC4 also mildly increases the level of apoptosis (Additional file 1: Fig. S3f).

To better characterize this senescent program, the transcriptomes of four LMS $\mathrm{HDAC4}^{-/-}$clones were compared with two control clones (expressing Cas9 or Cas9/ sgRNA1 but in which the KO was not achieved). The principal-component analysis is shown in Fig. S3g (Additional file 1). By applying stringent statistical criteria, we identified a minimal signature of 230 genes significantly modulated in all $H D A C 4^{-/-}$clones (Fig. 3d). One hundred forty-two out of 230 of these genes (62\%) are induced after HDAC4 deletion (Fig. 3e). An unbiased GSEA analysis identified a senescence geneset among the most enriched in $H D A C 4^{-/-}$cells (Fig. 3f). Moreover, senescence-associated secretory phenotype (SASP), RIS, and NFKB1 target genes are all positively enriched in $H D A C 4^{-/-}$cells (Fig. 3f).

To unambiguously prove that the CRISPR-mediated knock-out of HDAC4 is the primary cause of senescence, we re-expressed a Cas9 resistant mutant of HDAC4 $\left(H D A C 4^{P A M}\right)$ (Fig. 3g). This expression rescues the senescent phenotype in all clones (Fig. 3h), stimulates the entering into the cell cycle (Fig. 3i), and supports their growth in soft agar (Additional file 1: Fig. S3h). As expected, NFKB inhibition only partially recovers the senescent phenotype in terms of SA- $\beta$-gal positivity and progression through S-phase (Fig. 3j/k) [36].

Finally, the re-expression of HDAC4 in SK-LMS-1/HDAC4 ${ }^{-/}$cells rescues the expression of a set of genes, which define the SASP and the cell cycle arrest. Differently, blocking NFKB affects only the SASP genes and not the cell cycle related genes (Fig. 3l).

As the last model of senescence, we knocked-out HDAC4 in A375 melanoma cells. Melanoma was chosen since senescence barrier disruption during melanomagenesis is well reported [37]. Moreover, high levels of HDAC4 negatively correlate with patients' survival (Additional file 1: Fig. S4a). Initially, we obtained only heterozygous clones, suggesting an important role of HDAC4 in regulating cell fitness. Therefore, we generated (Doxycyline) DOX-inducible cell lines to conditionally express $H D A C 4^{P A M}$ before its targeting. With this strategy, five $H_{D A C 4^{-/-}}(320,304,401,150,1090)$ and two 


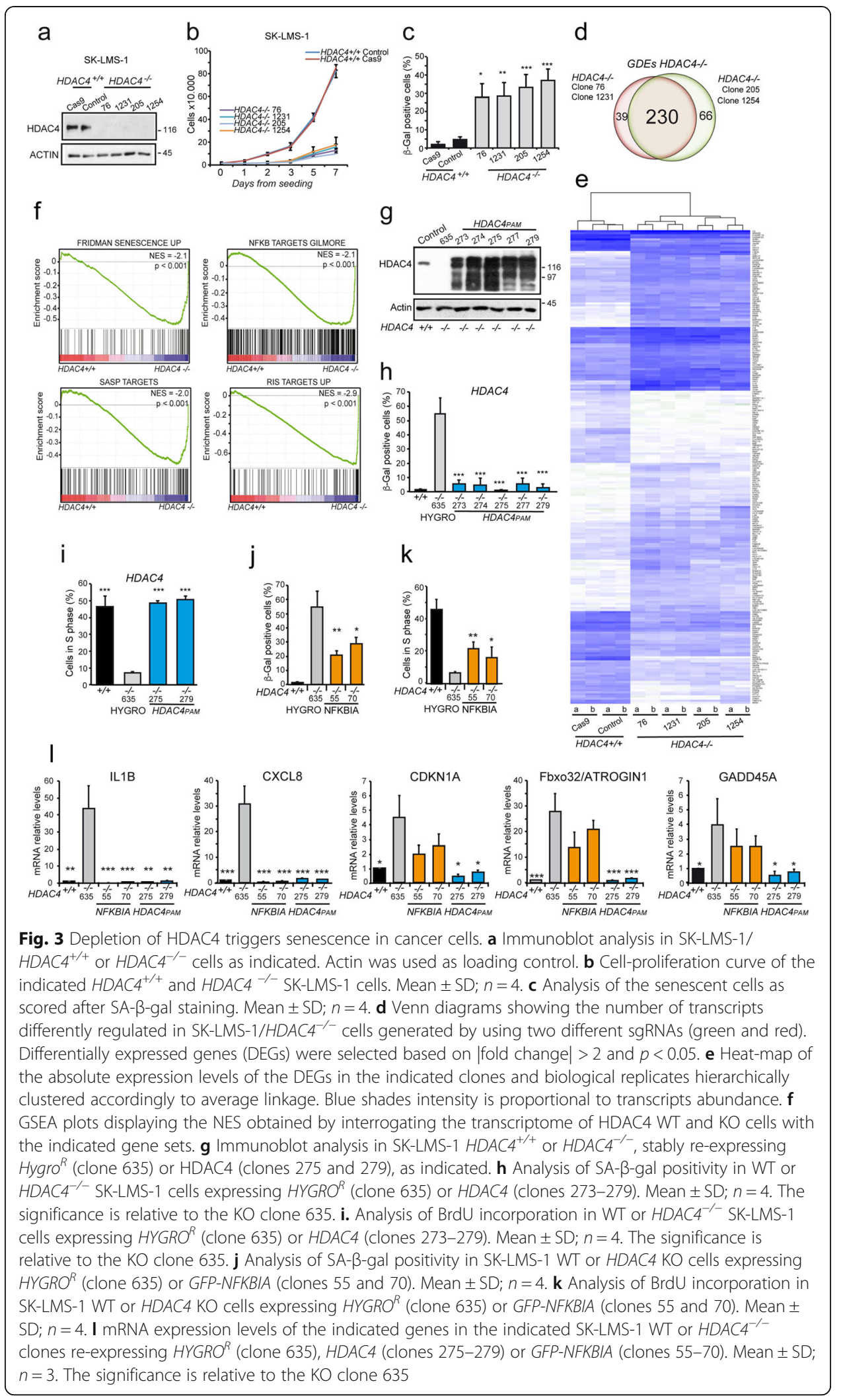


$H D A C 4^{+/-}$clones $(159,317)$ were isolated (Additional file 1: Fig. S4b). Similarly to LMS cells, A375/HDAC4 ${ }^{-/-}$cells evidence the downregulation of LMNB1 (Additional file 1: Fig. S4b/f) and the acquisition of classical senescent markers like cell cycle arrest (Additional file 1: Fig. S4c), SA- $\beta$-gal positivity (Additional file 1: Fig. S4d), and the appearance of cytosolic chromatin fragments (Additional file 1: Fig. S4e). All these phenotypes are weaker in the heterozygous $\left(\mathrm{HDAC4}^{+/-}\right)$clones and the re-expression of HDAC4 recovers the normal phenotype. The above-described genes repressed by HDAC4 in LMS cells turned out to be upregulated also in A375 $\mathrm{HDAC4}^{-/-}$cells (Additional file 1: Fig. S4g). The role of HDAC4 in antagonizing senescence in melanomas was confirmed by RNAi mediated silencing in WM115 cells (Additional file 1: Fig. S4h). LMNB1 downregulation, TP53 upregulation (Additional file 1: Fig. S4h), increased SA- $\beta$-gal positivity (Additional file 1: Fig. S4i), and the upregulation of a pool of senescent-related genes regulated by HDAC4 in LMS cells (Additional file 1: Fig. S4J) were all observed after HDAC4 silencing.

\section{The epigenome of $\mathrm{HDAC4}^{-/-}$cells is altered at the level of specific loci}

HDAC4 is an epigenetic regulator that supervises H3K27ac levels, particularly at distal regulatory regions [15, 38]. We hypothesized that HDAC4 could safeguard the epigenetic identity to counteract senescence. Having proved the role of HDAC4 in different models of OIS, we decided to investigate more in detail the epigenetic changes in LMS cells. This choice is justified by the following: (i) the role of class IIa and of their partners in this tumor is well characterized [15, 34, 39] and (ii) mutations in two key elements of the senescent pathway, $R B 1$ and CDKN2A, in LMS are mutually exclusive and strongly associate with patient survival (Fig. S5ab). To evaluate the epigenomic influence of HDAC4 depletion during the initial steps of senescence, we generated SK-LMS-1 $H D A C 4^{-/-}$cells that re-express a 4OHT inducible HDAC4, HDAC4 $4^{-/-} / H D A C 4^{P A M}-E R$ (Additional file 1: Fig. S5c). The tamoxifen inducible re-expression of HDAC4 in SK-LMS-1/HDAC4 ${ }^{-/}$cells (clone 66, +4-OHT) impairs the appearance of senescence, which can be quickly established following removal of 4-OHT. This effect was proved by the abrogation of SA- $\beta$-gal positivity (Additional file 1 : Fig. S5d), by the restoration of LMNB1 levels (Additional file 1: Fig. S5c) and cell proliferation (Additional file 1: Fig. S5e) and by the growth in soft-agar (Additional file 1: Fig. S5f). Moreover, the reexpression of HDAC4 abrogates two typical features of senescent cells: the sensitivity to the senolytic drug navitoclax/ABT-263 [40] (Additional file 1: Fig. S5g) and the loss of the linker histone H1 [41] (Additional file 1: Fig. S5h).

Next, ChIP-seq was performed in $H D A C 4^{-/} H D A C 4^{P A M}-E R$ cells at $36 \mathrm{~h}$ from the removal of 4-OHT, to monitor H3K27ac and H3K27me3 variations. Genome-wide analysis shows locus specific increases of H3K27ac levels in cells knocked-out for HDAC4. These loci do not show overt variations in H3K27me3 (Fig. 4a/b). In parallel, HDAC4 depletion triggers moderate decreases of H3K27me3 levels in loci that do not show overt variations in H3K27 acetylation (Additional file 1: Fig. S6a/b). The re-acetylation observed in $\mathrm{HDAC4}^{-/-}$cells involved mainly intronic (18\%) and intergenic regions $(45 \%)$, as similarly observed in other studies [15-17]. Finally, the reasons for H3K27me3 increase in some loci remain to be tested. 


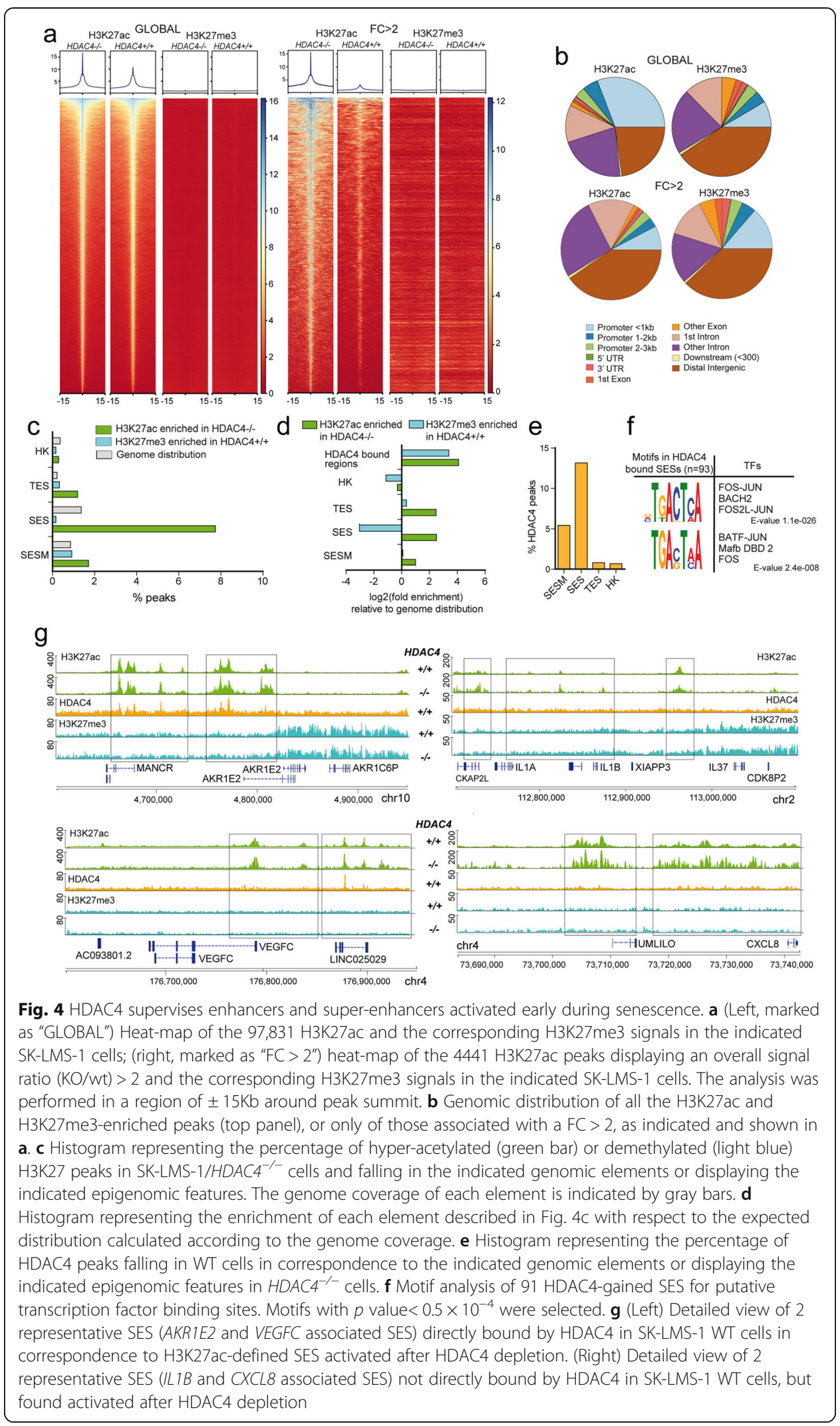


The enrichment for epigenetic modifications in intergenic regions upon HDAC4 depletion leads us to hypothesize that HDAC4 could monitor distal regulatory elements such as typical enhancers (TE) and super-enhancers (SE) associated with senescence. SE are large clusters of TE that are densely occupied by transcription factors and coregulators of transcription [42]. Hence, we defined an atlas of activated TE and SE that characterize cellular senescence by joining H3K27ac peaks enriched during replicative senescence [5] and OIS [4] (Additional file 2, Table S1). We defined them as TES (typical enhancers of senescence) and SES (super-enhancers of senescence), in respect to SESM (super-enhancers of smooth muscles) that typify this cell lineage. Two percent and $8 \%$ of the $\mathrm{H} 3 \mathrm{~K} 27 \mathrm{ac}$ regions hyper-acetylated in $\mathrm{HDAC4}^{-/-}$cells are found respectively in TES and SES (Fig. 4c).

\section{HDAC4 supervises enhancers and super-enhancers activated early during senescence}

The previous analysis suggests that hyper-acetylated $\mathrm{H} 3 \mathrm{~K} 27$ regions in $H D A C 4^{-/-}$cells are enriched for TES and SES. Hence, we hypothesized that HDAC4 could directly control H3K27ac levels at TES and SES. To prove it, we used ChIP-seq to map the genomic regions bound by HDAC4. Figure $4 \mathrm{~d}$ shows that HDAC4 binding in WT cells is enriched in correspondence of loci characterized by H3K27ac hyper-acetylation and H3K27me3 de-methylation after HDAC4 KO. This evidence suggests a direct involvement of HDAC4 in these epigenetic regulations, as further confirmed by the heatmap analysis for H3K27ac at SES (Additional file 1: Fig. S6c). In summary, 13.5\% of HDAC4 peaks colocalize with SES (Fig. 4e). HDAC4-bounded chromatin regions, which organize in SES after HDAC4 depletion, are characterized by a strong consensus for Activator Protein 1 (AP-1) (Fig. 4f). Figure 4g exemplifies 4 different loci containing SES, which H3K27ac levels increase after HDAC4 deletion. AKR1E2 and VEGFC are two loci containing the AP-1 consensus and characterized by HDAC4 recruitment (Fig. 4g left panel). By contrast, NFKB consensus characterizes SES not directly bound by HDAC4 (Fig. 4g right panel and Additional file 3, Additional file 3: Table S2), as exemplified by the ILIB and CXCL8 loci. The quantitative analysis confirmed an augmentation in H3K27 acetylation at these SES upon HDAC4 depletion (Additional file 4: Table S3). The activation of the TE that controls CDKN1A expression in HDAC4 KO cells is shown in Additional file 1: Fig. S6d.

HDAC4 is required to load a repressive complex in correspondence to well-defined AP-1 regulated SES and antagonizes $\mathrm{p} 300$

H3K27ac peaks modulated by HDAC4 can be found in 256 SES defined in other cell lines. Among them, 91 SES are directly regulated by HDAC4 and 63 of them bear the AP-1 consensus in correspondence to HDAC4 binding sites (Fig. 5a). Since SE can be cell type specific, to strengthen the importance of HDAC4 in the regulation of SES, ChIP-seq experiments were performed to map H3K4me1 distribution in SK-LMS-1 WT cells or at $36 \mathrm{~h}$ from HDAC4 depletion. Using the ROSE algorithm, 980 SE were globally identified in $\mathrm{HDAC4}^{-/-}$cells (Additional file 5: Table S4). Thirty-nine percent of them were previously defined as SES in other cell lineages (Fig. 5a), and 363 were specific of $\mathrm{HDAC4}^{-/-}$cells. This group of SE represents the SE induced during the early phases of senescence ( $36 \mathrm{~h}$ from HDAC4 depletion) in SK-LMS-1 cells. Twenty- 


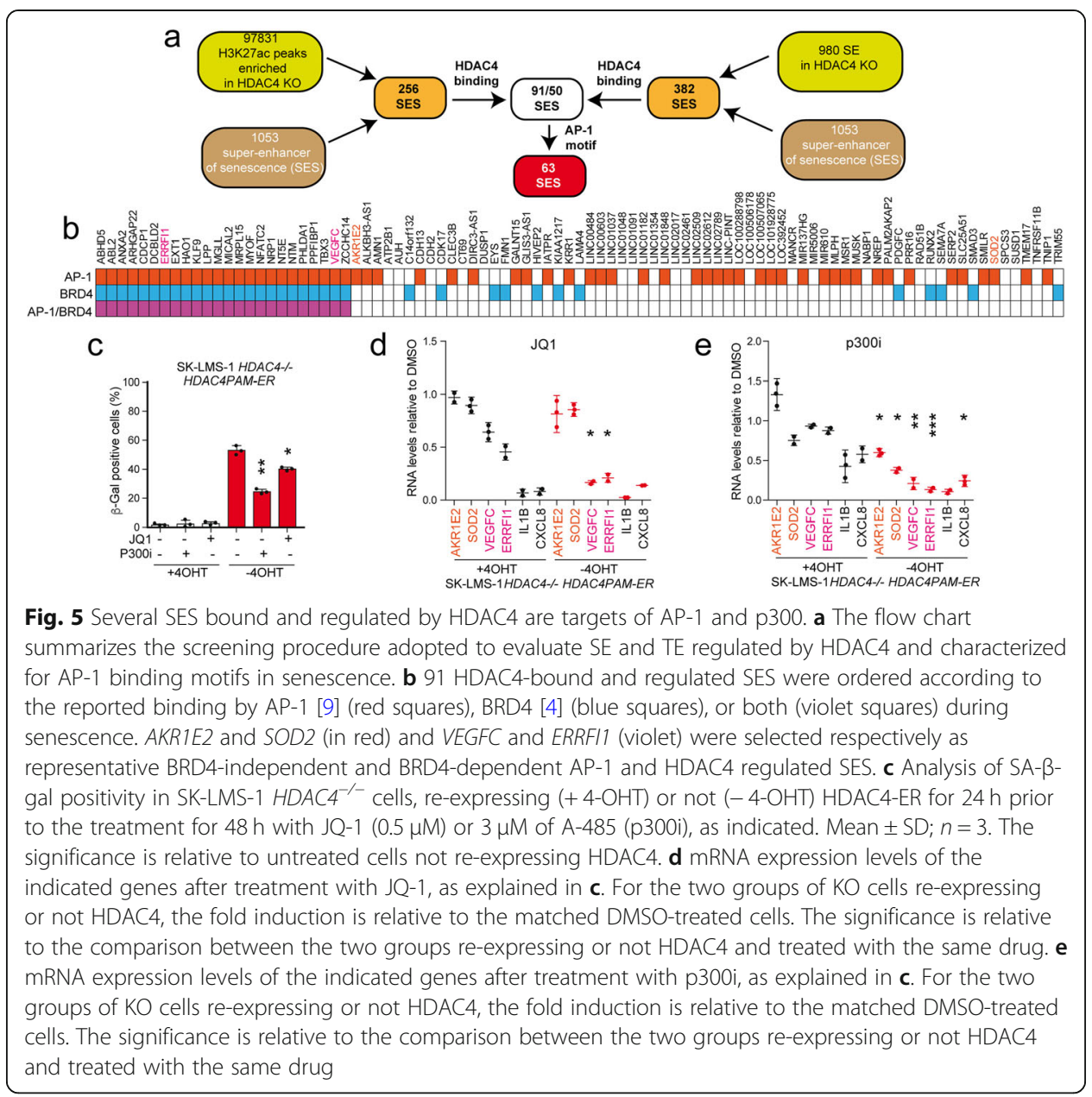

seven of these SES are marked by HDAC4 binding. The heat maps and the metaplots for H3K27ac levels in these SES are illustrated in the Additional file 1: Fig. S6e. Relying on the importance of HDAC4 in controlling SES dynamics, we next explored the role of AP-1 in the modulation of SES under HDAC4 influence. We focused the analysis on the 63 SES that bear the AP-1 binding sites and show variations in H3K27 acetylation. One third of these AP-1 regulated loci were found to be bound by BRD4 during senescence [4] (Fig. 5b). Together with BRD4, the HAT p300 sustains most of the transcriptional activities of AP-1 [43]. BRD4 is a mediator of transcription that densely occupies SE to drive gene transcription [4]. Hence, we investigated the contribution of BRD4 and p300 in transcribing SES associated genes in $H D A C 4^{-/-}$cells. The treatment with the p300 inhibitor (p300i/A-485) better counteracts senescence in HDAC4-depleted cells, with respect to the BRD4 inhibitor JQ-1 (Fig. 5c). We confirmed the efficiency of the inhibitors by scoring the expression of 6 SES-associated genes, 4 directly regulated (ERRFI1, AKR1E2, SOD2, VEGFC) and 2 indirectly regulated by HDAC4 (ILB, CXCL8). Of these genes, 4 were predicted to be BRD4 regulated: VEGFC, ERRFI1, IL1B, and CXCL8 (Fig. 5b). As expected, JQ-1 reduces the expression of BRD4 regulated genes (VEGFC, ERRFI1, IL1B, CXCL8), both in WT and in HDAC4-depleted cells, much potently for $I L 1 B$ and CXCL8 (Fig. 5d). The effect is stronger in KO cells only for VEGFC and ERRFI1. Differently from JQ-1, A-485 less efficiently impacts on the transcription 
of the six genes in WT cells (only ILIB and CXCL8), while it strongly blunts their expression in HDAC4-depleted cells (Fig. 5e). Collectively, these results suggest that HDAC4 sculpts the chromatin by competing with $\mathrm{p} 300$. Its absence could favor the organization of the chromatin into super-enhancers at AP-1 binding sites.

\section{HDAC4 supervises the deacetylation of AP-1 regulated SES}

HDAC4 assembles in multiprotein complexes where the recruitment of other corepressors is critical to mediate its epigenetic influences. To gain insight on the mechanisms operated by HDAC4 to monitor TE and SE associated with senescence, we used the wt HDAC4 in comparison to the mutant HDAC4 ${ }^{\triangle 600}$-GFP, lacking the deacetylase domain (DAC). This domain is required to recruit HDAC3 [44]. We also introduced HDAC3-GFP in HDAC4 depleted cells, for comparison.

While the re-expression of full-length HDAC4-GFP completely rescues the proliferative deficit observed in HDAC4 depleted SK-LMS-1 cells, the re-expression of the mutant HDAC4 $4^{\triangle 600}$-GFP fails in escaping senescence (Fig. 6a). A partial recovery of senescence was observed when HDAC3 is introduced (Fig. 6a). This rescue is inversely correlated with AP-1 transcriptional activity, which is deeply triggered in HDAC4 depleted cells. AP-1 transcriptional activity is not affected after GFP or HDAC4 ${ }^{\triangle 600}$-GFP, but it is strongly impaired after HDAC4-GFP and, to a lesser extent, after HDAC3-GFP expression (Fig. 6b). These results find confirmation in the HDAC4-dependent levels of H3K27ac at AP-1 regulated SES-associated genes (Fig. 6c) and in the relative mRNAs levels (Fig. 6d). Particularly, the repression of SES-associated genes after HDAC3 overexpression is not successful for those loci, like AKR1E2, in which HDAC4 is required for the efficient loading of HDAC3 on the chromatin (Fig. 6e). Interestingly, both HDAC4-GFP and HDAC3-GFP fail to bind the SE associated with IL1B (Fig. 6e). Similarly, the expression of HDAC4 or E1A in BJ/hTERT/RAS cells blunts the upregulation of those SES-associated genes (Fig. $6 \mathrm{f}$ ) that are directly bound and regulated by HDAC4 (Fig. 6g). Again, IL1B and CXCL8 do not evidence any direct binding of HDAC4 and, more importantly, they are not repressed by HDAC4 or E1A, as a consequence of OIS escape (Fig. 6f). When normalization was calculated respect to input the results were identical, thus indicating that, within the time-frame of the experiment, $\mathrm{H} 3$ levels remain unperturbed. To summarize, HDAC4 controls the HDAC3-assisted deacetylation of a pool of AP-1-regulated SES whose de-repression is associated with senescence entrance and whose repression correlates with senescence escape.

\section{A local chromatin environment and not the binding qualities affects HDAC4 activity on well-defined SES}

HDAC4 and HDAC3 do not bind directly the DNA and are recruited at specific genomic loci by interacting with TFs as part of multiprotein complexes [45]. To further understand the impact of these HDACs on H3K27ac dynamics, we forced their localization to selected SES, independently from their ability to bind these regions in vivo. To this purpose, we delivered HDAC4 and HDAC3 fused to the dead mutant of Cas9 (dCas9 or Cas9 $9^{\mathrm{D} 10 \mathrm{~A}, \mathrm{H} 840 \mathrm{~A}}$ ) in BJ/hTERT cells expressing for 7 days $R A S$, together with 4 sgRNAs to re-direct dCas9-fused proteins on the selected loci (Fig. 7a). We chose $I L 1 B$ and $C C L X 8$, as two SES not recognized by HDAC4, as well as AREG 


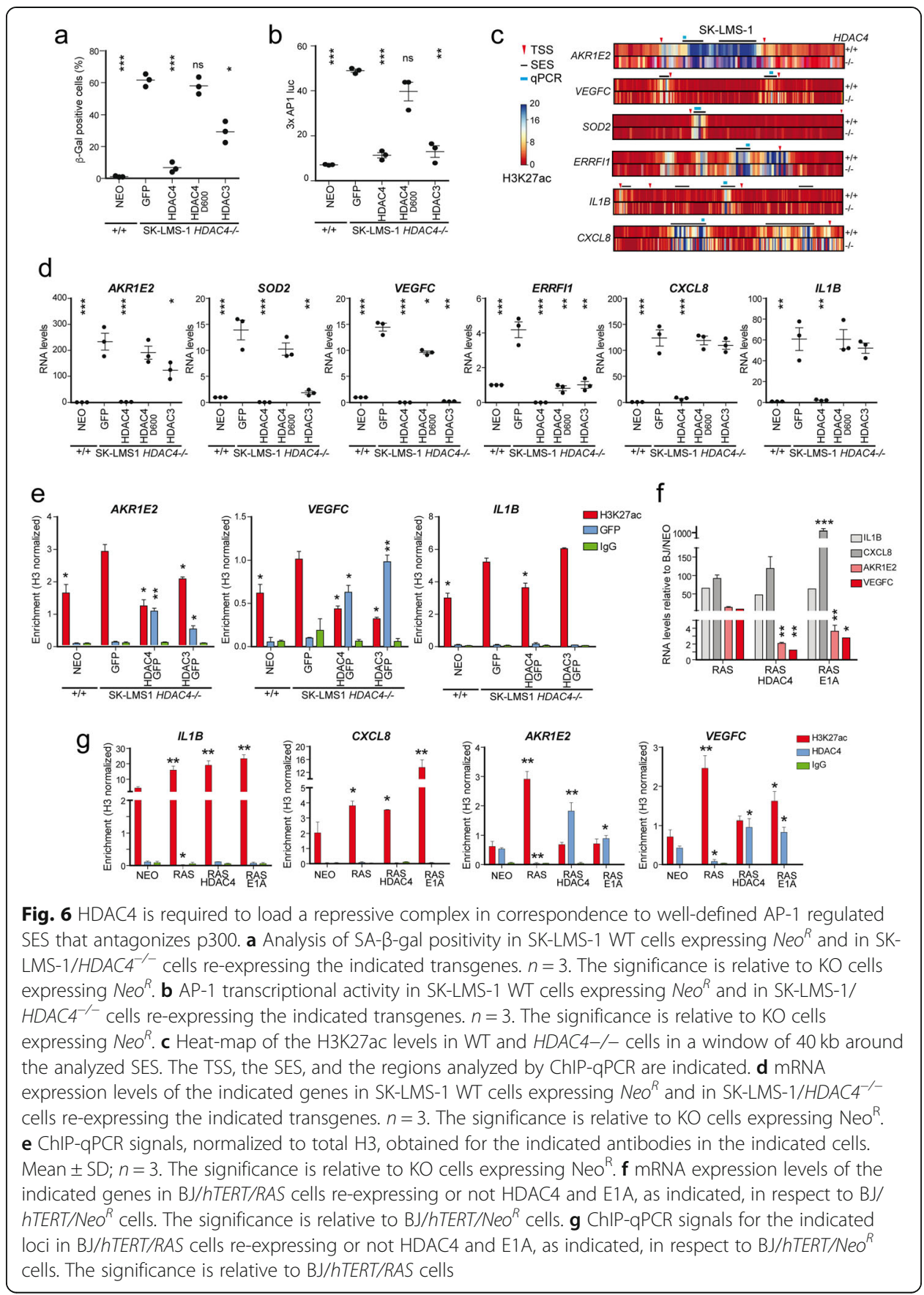

and SOD2, as examples of HDAC4-bound SES. ChIP experiments were performed $48 \mathrm{~h}$ later to evaluate both the binding of the different chimeras at the designed loci and H3K27ac levels. The Cas9 ChIP shows that the transfected sgRNAs are able to localize the different chimeras at the expected loci. Importantly, HDAC4 can partially, but substantially, re-direct the Cas9-chimera from IL1B/CCLX8 to SOD2/AREG superenhancers. This result indicates a strong and autonomous affinity of HDAC4 for these genomic regions (Fig. $7 \mathrm{~b}$ ). To exclude unspecific binding to DNA, we performed the same ChIP on distal genomic regions on the same chromosome. In this case, enrichments were not observed (Fig. 7c), thus confirming the specificity of the targeting. 


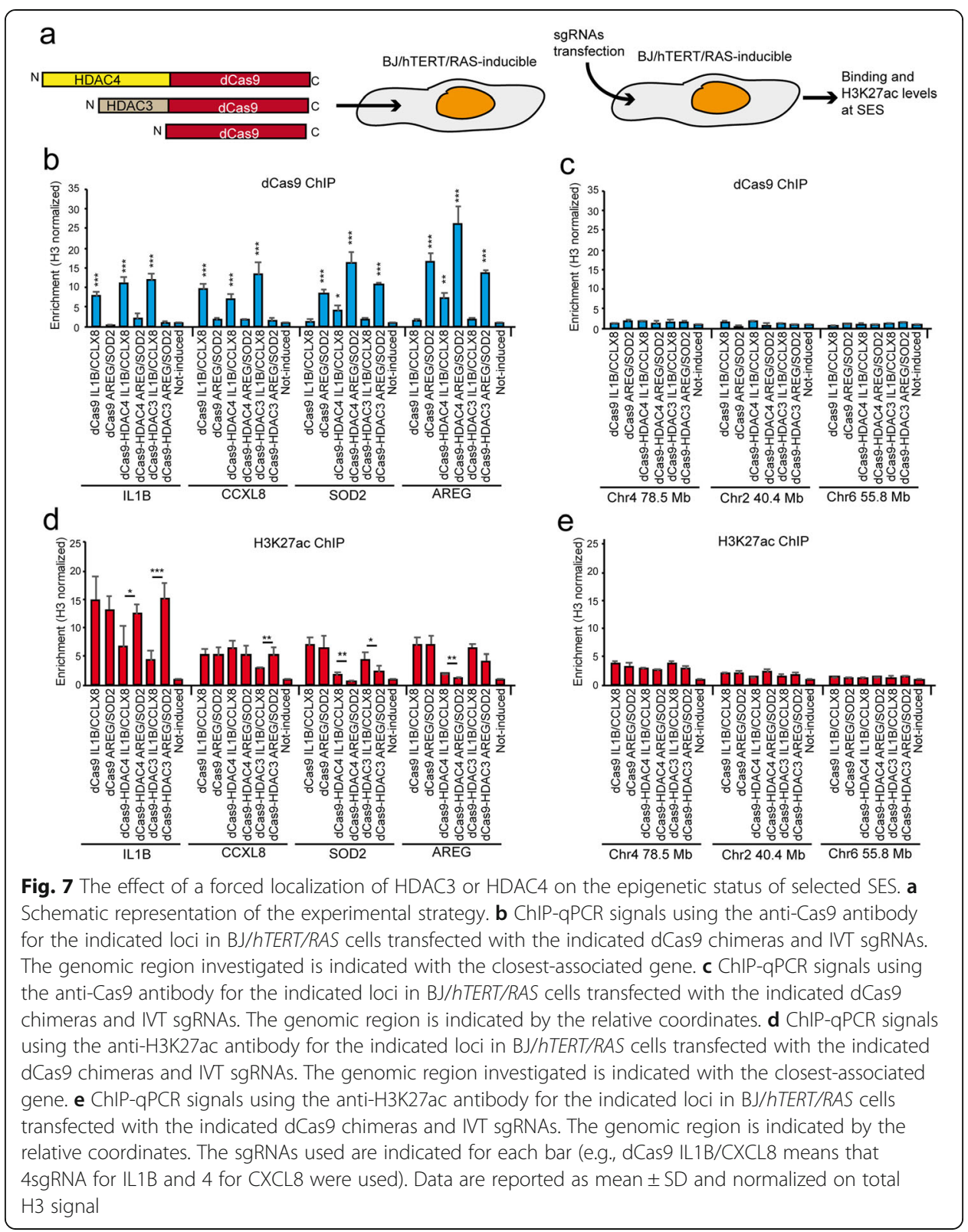

Next, we evaluated H3K27ac levels on the targeted regions. HDAC4-dCas9 chimera reduced the acetylation at AREG and SOD2 SES, whereas it was unable to act on CCLX8 SES and only partially able to influence IL1B SES, even though successfully targeted on these regions (Fig. 7c). By contrast, HDAC3-dCas9 is able to buffer H3K27ac at CXCL8 and IL1B super-enhancers, while it poorly deacetylates H3K27 on the AREG and SOD2 loci. In conclusion, these experiments show that HDAC4 has a strong affinity for the binding and the deacetylation of two HDAC4-specific SES (SOD2 and AREG associated SEs) that we have identified in this manuscript. The forced Cas9-dependent localization of HDAC4 on ILIB and CXCL8-associated SES leads to some deacetylation only in the case of IL1B. This means that the local chromatin environment at certain SES, like CXCL8, can impair not only the loading but also the activity of HDAC4 repressive 
complexes. On the opposite, the HDAC activity of HDAC3-dCas9 is relevant on $I L 1 B$ and CXCL8 SES, but it is marginal on the tested HDAC4-specific SES (SOD2, AREG), meaning that HDAC4 assists the activity of HDAC3 not only by directing its binding, but also by acting as recruiting factor for its local action on chromatin.

\section{Discussion}

Enhancer dynamics characterize the appearance of senescence $[4,5]$. These extended regions marked by H3K27ac, H3K4me1, and by clusters of transcription factor binding sites supervise the genetic program of senescent cells and particularly the SASP response during OIS [5]. HDAC4 is involved, directly or indirectly, in controlling the H3K27ac levels in approximately 25\% of previously defined SES. Moreover, in $9 \%$ of them, the direct binding of HDAC4 was observed. Although the list of SES was obtained from studies performed on other cell lines and lineage specific differences exist [14], these data are consistent with an important role of HDAC4 in buffering the fullactivation of TEs and SEs involved in senescence. In this scenario, it is important to note the dynamic nature of class IIa HDACs binding to chromatin [14]. A fast turnover of HDAC4 on chromatin could be an advantage to quickly induce the senescence response. Although dynamically involved in regulating different genomic regions, we have observed that the abrogation of HDAC4 acts rather as an epigenetic priming of senescence that only indirectly and as a later response gives rise to SASP. In fact, even though we forced the loading of HDAC4 onto CXCL8 and IL1B SES, the H3K27ac status was unperturbed or minimally perturbed (IL1B) by HDAC4, especially if the repressive effect is compared to its "natural" SES (AREG and SOD2). Therefore, some SES and TES could be indirectly controlled by HDAC4 in a hierarchical manner. HDAC4 and class IIa HDACs in general can form complexes with class I HDACs and with HDAC3 in particular [18]. We have observed that H3K27ac level at some SE can be modulated by both HDAC4 and HDAC3 whereas, at AREG SE, H3K27ac is modulated only by HDAC4. These preliminary observations deserve further investigations to precisely map the genomic regions bound by these two epigenetic regulators.

Among the SES bound by HDAC4, 66\% are enriched for the AP-1 binding motif. AP-1 groups different proteins that can homo- and heterodimerize to constitute the active TF. This complexity guarantees numerous combinations of this TF, which show different transcriptional outputs [46]. It is currently unknown which heterodimers bind and control SES. Nevertheless, our data figured out that HDAC4, assisted by HDAC3, supervises the chromatin status at AP-1 regulated SES, possibly by locally antagonizing p300 activities. In this context, AP-1 has been recently discovered to play relevant roles in pioneering and organizing SES $[4,9]$. During senescence entrance, the buffering activity of HDAC4 is lost, as a consequence of its controlled UPS-mediated and GSK3 $\beta$ assisted degradation. In this manner, TE and SE can orchestrate the transcriptional reprogramming. Certainly, the mechanisms engaged by HDAC4 to silence these regions deserve further characterizations and there are several possibilities. HDAC4 could, directly or indirectly, repress AP-1 mediated transcription or, through MEF2, it could also control JUN levels [34] (Additional file 1: Fig. S6f).

The critical role of HDAC4 in senescence is sustained by additional evidence. First, its depletion forces senescence entrance in transformed and pre-transformed cells of different lineages, such as melanomas and leiomyosarcomas. Second, the reintroduction 
of HDAC4 in a RAS-dependent model of OIS counteracts senescence more efficiently with respect to the inhibition of TP53.

Finally, although studies have identified HDAC4 as an epigenetic controller of senescence during OIS and RS, this discovery requires validation, particularly in those senescence responses that do not appear to be characterized by genomic instability, such as $\mathrm{NOTCH}$-induced senescence [47] and embryonic senescence [48].

\section{Conclusions}

Acquiring knowledge on the epigenetic regulation of senescence is fundamental to develop therapeutic approaches to target several age-related diseases including cancer. In summary, our screening aimed to depict class IIa HDACs regulation in senescence has unveiled a complex circuit of events that orbit around HDAC4. Whether the control operated by HDAC4 on the distal regulatory elements such as TE and SE is the sole action to counteracts OIS deserves further studies.

\section{Methods}

\section{Cell culture and reagents}

BJ/hTERT and IMR90 cells, SK-LMS-1 (TP53 $\left.{ }^{\mathrm{wt} / \mathrm{G} 245 \mathrm{~S}}\right)$ leiomyosarcoma cells (previously characterized in [15]), HEK293T, LinXE, Ampho Phoenix, and MEF HDAC4 $4^{\text {loxp/loxp }}$ cells were cultured as previously described [15] in 10\% FBS DMEM (Euroclone). A375 $\left(T P 53^{w t / w t}\right)$ and WM115 [49] (TP53 $\left.3^{w t / w t}\right)$ melanoma cells were grown in RPMI. For the conditioning of BJ/hTERT cells, the medium obtained from BJ/hTERT/HRAS $S^{G 12 V}$ or HYGRO cells cultured in $60 \mathrm{~mm}$ plates for 8 days was filtered and diluted 1:1 with fresh medium and used to treat cells twice for $96 \mathrm{~h}$, with a change after $48 \mathrm{~h}$. For the experiments performed in hypoxia, cells were grown in hypoxic chambers at $37^{\circ} \mathrm{C}, 5 \% \mathrm{CO}_{2}$, and $2 \% \mathrm{O}_{2}$ (Baker Ruskinn). The following chemicals were used: $250 \mathrm{nM}$ 4-OHT (Sigma-Aldrich), $1 \mu \mathrm{M}$ Doxycycline (Sigma-Aldrich), $1 \mu \mathrm{M}$ MG132 (Sigma-Aldrich), $10 \mu \mathrm{M}$ Chloroquine (Sigma-Aldrich), $50 \mathrm{ng} / \mathrm{ml}$ Leptomycin B (Sigma-Aldrich), 0.4\% Trypan Blue (Sigma-Aldrich), $200 \mu \mathrm{M} \quad \mathrm{H}_{2} \mathrm{O}_{2}$ (Sigma-Aldrich), $1 \mu \mathrm{M}$ PD0332991 (Sigma-Aldrich), $100 \mathrm{nM}$ ABT-263 (Clinisciences), $500 \mathrm{nM}$ JQ1 (Vinci Biochem), and $3 \mu \mathrm{M}$ A-485 (Vinci Biochem). For luciferase assay, SK-LMS-1 cells HDAC4 wt or KO re-expressing HDAC4 mutants or HDAC3 were transfected with 300 ng pGL3-3XAp1luc plasmid (Addgene \# 40342) and $30 \mathrm{ng}$ pTK-Renilla Luc plasmid. After $48 \mathrm{~h}$, cells were harvest and the assay was performed accordingly to Dual-Glo Luciferase Assay System (Promega). Luminescence was recorder by using Modulus Luminometer Microplate reader (Turner BioSystems) and Luciferase signal was normalized to Renilla signal.

\section{Generation and culture of $\mathrm{Hdac}_{4}^{\mathrm{flfl}}$ and $\mathrm{Hdac}^{-/-}$murine embryonic fibroblasts}

$H d a c 4^{f l f l}$ mice were previously described [50]. MEFs were generated following standard procedures [51] from 13.5 days-old embryos. Single cell suspensions were expanded in DMEM/10\% FBS in hypoxia. $5 \times 10^{6}$ cells were retrovirally infected to express Cre-ER. Not infected cells were removed from culture by puromycin $(2 \mu \mathrm{g} / \mathrm{ml}$, Sigma-Aldrich $)$ selection. The recombination was achieved through the treatment for $48 \mathrm{~h}$ with 4-OHT (Sigma-Aldrich). At the end of this incubation, half of the culture was kept in normoxia 
and half in hypoxia. At each splitting, the total number of cells was counted (Countess II, LifeTechnologies), and the doubling time (dt) was calculated as previously described [52].

Plasmid construction, transfection, retroviral and lentiviral infection, and silencing pLENTI-CRISPR/V2 (Plasmid \#52961), pSpCas9(BB)-2A-GFP (PX458) (Plasmid \#48138), pSpCas9(BB)-2A-Puro (PX459) (Plasmid \#62988), pCW-Cas9 (Plasmid \#50661), pCW57/Hygro-MCS1-2A-MCS2 (Plasmid \#80922), pBabe-Puro-IKBalpha (NFKBIA) S32A/S36A (Plasmid \#15291), MSCV-CreERT2-Puro (Plasmid \#22776), and dCas9-hHDAC3 (Plasmid \#98591) were obtained from Addgene. pWZL-Hygro$H D A C 4^{P A M}$, pWZL-Hygro-HDAC4 ${ }^{P A M}-E R$, and $\mathrm{pCW}-H y g r o-H D A C 4^{P A M}$ were obtained by sub-cloning a mutagenized HDAC4 (QuikChange Site-Directed Mutagenesis Kit, Agilent) into the linearized empty backbones by a restriction-based approach. pWZLHygro-ER acceptor plasmid was previously described [53]. Apple-53BP1trunc and $\mathrm{H2B}$ GFP were sub-cloned respectively in pBABE-Zeo and pWZL-Neo, NFKBIA-S32A/S36A into pWZL-Neo-GFP. pWZL-Neo-MCS1-2A-MCS2 was obtained by sub-cloning the MCS of pCW57 Hygro MCS1-2A-MCS2 into pWZL-Neo through a recombinationbased approach. The generated plasmid was used as acceptor vector for the cloning of $H_{R A S}{ }^{G 12 V}$ (NheI/SalI) and E1A/1-143 (MluI/BglII-BamHI) to generate pWZL-Neo$H_{R A S}{ }^{G 12 V}-2 \mathrm{~A}-E 1 A$ 1-143. pWZL-Hygro-HRAS ${ }^{G 12 V}$, pBABE-Puro-HRAS ${ }^{G 12 V}$, and pBABE-Puro-myrAKT1 were previously described [32, 54]. pWZL-Neo/HDAC4-GFP, pWZL-Neo/HDAC4 ${ }^{\triangle 600}, \quad$ pWZL-Neo/HDAC3-GFP, pWZL-Neo/HDAC4-3xFLAG, pWZL-Neo/ dCas9 NLS, pWZL-Neo/HDAC4-dCas9 NLS, pWZL-Neo/HDAC3-dCas9 NLS, pCW-Puro/HRAS ${ }^{G 12 V}$, and myrAKT1 were obtained by sucloning. All the generated plasmids were checked by restriction and sequencing. The primers used for cloning are listed in additional file 4 Table S3. HDAC4 ${ }^{\text {PAM }}$ (HDAC4 CDS NM_006037) bears point mutations $(\mathrm{V} 31 \mathrm{~L})$ in correspondence to the PAM to prevent its targeting by Cas9. Its efficacy similar to the WT form has been tested on the repression of the target KLF2 and on the formation of the complex with MEF2D [34].

Transfections, viral infections, and siRNA delivery were done as previously described $[17,54]$. The following siRNAs (148 pmol) were used: HDAC4 (CCACCGGAAUCUGA ACCACUGCAUU, Invitrogen Stealth) and scramble control siRNA (UAAGGCUAUGAAGAGAUA, Invitrogen Stealth).

\section{CRISPR/Cas9 genome editing}

SpCas9 was stably transduced to generate SK-LMS1 $H_{D A C 4^{-/-}}$clones $(76,1231,205$, 1254,66 ), and BJ/E1A-RAS $H D A C 4^{-/}$. SpCas9 was transiently transfected (Lipofectamine 2000, LifeTechnologies) to generate SK-LMS-1 $H D A C 4^{-/-}$clones $(635,273,274$, 275, 277, 279), A375 $\mathrm{HDAC4}^{-/-}$clones $(304,401,150,1090)$, and $\mathrm{HDAC4}^{+/-}$clones $(317,159)$. The Cas9-resistant HDAC4 (HDAC4 ${ }^{\mathrm{PAM}}$ ) was stably expressed prior to the KO (in SK-LMS-1 clones $273,274,275,277,279$ ) or continuously re-expressed in a 4OHT-dependent (SK-LMS-1 clone 66) or DOX-dependent (A375 clones 304, 401, 150, 1090) manner, similarly to what previously described [17]. The sgRNA used are listed in additional file 4 Table S3. Monoclonal cultures were generated by seeding $n=1$ (SKLMS-1 and A375) and $n=3$ (BJ/E1A-RAS) cells in each well of 96-well plates (Sarstedt). 
The successful generation of $\mathrm{KO}$ clones was screened by immunoblotting and confirmed by Sanger sequencing.

\section{Design and delivery of sgRNAs and dCas9 chimeras}

Four sgRNAs for the targeting of each SEs were in vitro transcribed (IVT) according to GeneArt $^{\text {tw }}$ Precision gRNA Synthesis Kit (Invitrogen). IVT products were treated prior to purification with $5 \mathrm{U}$ of DNAseI (Fermentas) and $10 \mathrm{U}$ of CIP (New England Biolabs), respectively to destroy DNA templates and blunt the dsRNAs inflammatory response [55]. $15 \times 10^{6} \mathrm{BJ} / \mathrm{hTERT}$ cells expressing RAS were lipofected (Lipofectamine 3000, Thermo Fisher Scientific) with $8 \mu \mathrm{g}$ of plasmidic DNA encoding Cas9-fused proteins and $8 \mu \mathrm{g}$ of IVT-treated sgRNAs ( $2 \mu \mathrm{g}$ for each sgRNA). Two days later, chromatin was crosslinked, extracted, quantified, and processed for ChIP. The sequence of the sgRNAs used is provided in additional file 4 Table S3.

\section{Immunofluorescence and immunoblotting}

Cells were fixed with 3\% paraformaldehyde and permeabilized with $0.3 \%$ Triton X-100. The secondary antibodies were Alexa Fluor 488-, 546-, or 633-conjugated anti-mouse and anti-rabbit secondary antibodies (Molecular Probes). Actin was labeled with phalloidin-AF546 or AF-660 (Molecular Probes). For S phase analysis, cells were grown for $3 \mathrm{~h}$ with $50 \mu \mathrm{M}$ Bromodeoxyuridine (BrdU). After fixation, coverslips were treated with $\mathrm{HCl}(1 \%$ and $2 \%)$, quenched with Borate, and processed for immunofluorescence. Cells were imaged with a confocal microscope Leica AOBS SP8 or with Leica AF6000 LX. Nuclei were stained with Hoechst 33258.

Cell lysates after SDS-PAGE and immunoblotting on nitrocellulose (Whatman) were incubated with primary antibodies. HPR-conjugated secondary antibodies were obtained from Cell Signaling, and blots were developed with Super Signal West Dura (Thermo Fisher Scientific). Primary and secondary antibodies were removed by using Restore PLUS Western Blot Stripping Buffer (Thermo Fisher Scientific), according to the manufacturer. Unless otherwise indicated, all the immunoblot figures were representative of at least two biological replicates. The primary and secondary antibodies used in this work are listed in the additional file 7, Table S6.

\section{Proteomics and transcriptomics from in vivo murine aging models}

C57BL/6 J female mice were obtained from Shared Ageing Research Models (ShARM, UK). Tissues explanted from 4 months (128 days) and 26 months (774 days) old mice were snap-frozen in liquid nitrogen. For protein lysates generation, subsections of the liver and of the skin were grinded into a powder with a pestle and lysed for $1 \mathrm{~h}$ at $4{ }^{\circ} \mathrm{C}$ respectively with 400 and $200 \mu \mathrm{L}$ RIPA lysis buffer for $10 \mathrm{mg}$ of tissue. $4 \mathrm{x}$ Laemmli sample buffer was added to the clarified lysates and after boiling the samples were loaded on SDS/PAGE gels. For RNA extraction, $1 \mathrm{ml}$ Tri Reagent (Molecular Research Center) was added to $10 \mathrm{mg}$ of smashed tissues. After $1 \mathrm{~h}$ incubation at $4{ }^{\circ} \mathrm{C}$, RNA was recovered by phenol-chloroform extraction/ethanol precipitation and resuspended in $20 \mu \mathrm{L}$ RNase-free water. 


\section{Immunoprecipitation}

Cells were lysed for 10' into hypotonic lysis buffer $(20 \mathrm{mM}$ Tris- $\mathrm{HCl} \mathrm{pH} \mathrm{7.4,10} \mathrm{mM} \mathrm{KCl,}$ $10 \mathrm{mM} \mathrm{MgCl} 2,1 \%$ Triton X-100, 10\% glycerol, $50 \mathrm{mM}$ Iac, $1 \mathrm{mM}$ phenylmethylsulfonyl fluoride, $5 \mathrm{mM} \mathrm{NaF}, 1 \mathrm{mM} \mathrm{Na} \mathrm{VO}_{4}$ ), supplemented with protease inhibitors and $10 \mu \mathrm{M}$ MG-132 and $10 \mu \mathrm{M}$ G5. Lysates were incubated for $5 \mathrm{~h}$ with $1 \mu \mathrm{g}$ anti-HDAC4 [56] or rabbit IgG and for $1 \mathrm{~h}$ with $30 \mu \mathrm{L}$ slurry protein A (GE). After 4 washes, the immunocomplexes were reversed with 2x Laemmli sample buffer, boiled, resolved by SDS-PAGE, and subjected to western-blotting. One of 100 of total lysate has been collected as input.

\section{SA- $\beta$-gal assay}

Cells seeded on coverslips in 12-well plates were fixed for $5^{\prime}$ (PBS $2 \%$ formaldehyde/ $0.2 \%$ glutaraldehyde), washed twice with $0.9 \% \mathrm{NaCl}$, and stained for $16 \mathrm{~h}$ at $37^{\circ} \mathrm{C}$ with staining solution: $40 \mathrm{mM}$ citric acid/Na phosphate buffer, $5 \mathrm{mM} \mathrm{K4}[\mathrm{Fe}(\mathrm{CN}) 6] 3 \mathrm{H} 2 \mathrm{O}, 5$ $\mathrm{mM} \mathrm{K3}[\mathrm{Fe}(\mathrm{CN}) 6], 150 \mathrm{mM}$ sodium chloride, $2 \mathrm{mM}$ magnesium chloride, and $1 \mathrm{mg} / \mathrm{mL}$ $\mathrm{X}$-gal (Panreact Applichem). Images were acquired with Leica LD bright field optical microscope.

\section{Transformation assay}

Soft agar assay was performed as previously described [32]. Briefly, a total of $0.8 \times 10^{5}$ cells were seeded in $0.3 \%$ top agar/DMEM layer above a 0.6\% agar/DMEM basement. Fresh medium was added twice/week. After 15 days of culture, the supernatant was discarded and the MTT [3-(4,5-dimethylthiazol-2-yl)-2,5-diphenyltetrazolium bromide] staining $(0.5 \mathrm{mg} / \mathrm{ml}$ in PBS) was applied for $2 \mathrm{~h}$. Images were acquired with a Leica DN6000 microscope. Foci were automatically counted with Clono Counter.

\section{RNA extraction and quantitative qRT-PCR}

Cells were lysed using Tri Reagent (Molecular Research Center). $1.0 \mu \mathrm{g}$ of total RNA was DNAseI treated (NEB \#T2010) and retro-transcribed by using 100 units of M-MLV Reverse transcriptase (Life Technologies) in the presence of $1.6 \mu \mathrm{M}$ oligo (dT) (SigmaAldrich) and $4 \mu \mathrm{M}$ Random hexamers (Euroclone). qRT-PCRs were performed using SYBR green technology (KAPA Biosystems). Data were analyzed by comparative threshold cycle (delta delta $\mathrm{Ct}$ ) using HPRT and GAPDH or ACTB and GAPDH as normalizer. The primers used for qRT-PCR are listed in the additional file 6 Table S5.

\section{RNA array expression and data analysis}

Total RNA was purified with Quick-RNA Miniprep (ZymoResearch), amplified according to the specifications of the Illumina TotalPrep RNA Amplification Kit (Ambion) and hybridized on Illumina whole-genome HumanHT-12 v 4.0 chip (Illumina). Acquisition and data analysis were performed as previously described [15]. Principal component analysis (PCA) was performed by using $\mathrm{R}$ function prcomp. Differentially expressed genes (DEGs) were called accordingly to the following criteria: |fold change $\mid>2$ and $\mathrm{p}$ adj. $<0.05$. GSEA analysis in Fig. 2 was performed as previously described [15]. The transcripts defining the "NFk $\beta$," "SASP" [57], and "RIS upregulated genes" gene sets are listed in the additional file 8, Table S7. Gene list enrichment was performed by interrogating MSigDB collections (BP,C6,CGP,H,MF) with the transcripts 
associated to promoters (with $2 \mathrm{~kb}$ from TSS), TEs or SEs bound by HDAC4; the obtained enrichments were considered significant for $\mathrm{p}$ and FDR $<0.05$ and if at least three Gene Sets fall in the same category. For the expression levels and Kaplan-Meier analysis of TCGA Skin Cutaneous Melanoma samples, data were retrieved from CBioPortal [58] and expressed as $z$-score. $Z$-scores $>|1.75|$ were selected as cutoff. For bioinformatics analysis in Fig. 3, the following GEO datasets were analyzed: GSE38410, GSE74324, GSE40349, GSE3189, GSE78138, GSE45276, GSE36640, GSE40349, and GSE132569.

\section{ChIP, library construction, ChIP-seq, and NGS data analysis}

Chromatin was obtained from SK-LMS-1 cells, $36 \mathrm{~h}$ after or not HDAC4 removal, and immunoprecipitated with $2 \mu \mathrm{g}$ of anti-H3K27ac, $3 \mu \mathrm{g}$ of anti-H3K27me3, $4 \mu \mathrm{g}$ of antiGFP, $3 \mu \mathrm{g}$ of anti-H3, and $4 \mu \mathrm{g}$ of anti-HDAC4 antibodies or control IgG, as previously described [15]. Three independent biological replicates were pulled according to BLUEPRINT requirements and $5 \mathrm{ng}$ of total DNA were used to prepare ChIP-seq libraries, according to TruSeq ChIP Sample Preparation guide (Illumina). Libraries were sequenced on the Illumina HiSeq 2000 sequencer. A minimum overlap of $27 \%$ of peaks between independent replicates was obtained and verified before the pulling. The ShortRead R/Bioconductor package was used to evaluate the quality of sequencing reads and Bowtie 2 was used to align them to NCBI GRCh38 human genome reference. Peak calling was performed against input sequences using HOMER for HDAC4 ChIP ("factor" mode) and MACS2 for H3K27ac and H3K27me3 ("sharp" mode and "broad" mode, respectively); gene annotations were performed as previously described [15]. gplots, biomaRt, and Gviz R/Bioconductor packages and the deepTools suite were used to generate peak heatmaps and for the visualization of genomic loci. We used the MAnorm method [59] to compare the ChIP-seq signals in the HDAC4 WT and KO conditions. For H3K27ac, H3K27me3, and H3K4me1 signals, the pre- and postnormalization regression lines were very similar and did not require further normalization. For the qPCR-ChIP experiments, normalization was against total H3. Under the time of the analysis $\mathrm{H} 3$ protein levels were not decreased. A decrease in $\mathrm{H} 3$ was observed only at much later times from HDAC4 depletion-induced senescence.

The H3K27ac and H3K27me3 enriched genomic regions between HDAC4 KO and wt were called according to $\sum_{k=\text { peak summit }}^{ \pm 15000} f(k)$, where $f(k)=\frac{\text { enrichment } K O}{\text { enrichment } w t} \cdot\left|\log _{2}(\mathrm{Fc})\right| \geq 1$ was used as cutoff. As a reference of smooth muscle super-enhancer, the sample_01_ 066_SE of smooth muscle SE retrieved from the SEdb [60] database (http://www. licpathway.net/sedb/) was used. "SES," defined according to the ROSE algorithm [61], represents the SEs activated during senescence. SES is the union of the SE identified during OIS [4] and replicative senescence [5] (Additional file 2 Table S1). Closest active algorithm was used for the association between genes and SEs. Liftover tool was used to convert genome coordinates between assemblies and to remap homologous sequences between genomes. The bedtools toolset (--intersect option) [62] was used to identify overlaps of at least one nucleotide between H3K27ac and HDAC4 peaks and the identified SEs and TEs. The investigated classes of genomic elements have been considered as separated, not redundant and not overlapping. The enrichment has been calculated with respect to the genome coverage of each genomic element (length of female 
haploid human genome: 3184709445 nucleotides). Known and novel motif discovery was performed using the MEME-ChIP tool from the MEME Suite [63]. The following parameters were used: -ccut 0 ; -order 1 ; -meme-maxsize 100000000; -meme-minsites 2; -mememaxsites 100; -mememinw 6; -meme-maxw 10; -meme-nmotifs 10; -meme-mod anr; -dreme-e 0.05 ; -centrimo-score 5.0 ; -centrimo-ethresh. Using a 1-order background model to normalize for biased distribution of letters and groups of letters in the analyzed sequences allows to specifically adjust the background for dimer biases (e.g., GC content). The identified enriched motifs were compared to the Jolma2013, JASPAR2018_CORE_ vertebrates_non_redundant and uniprobe_mouse databases for annotation. Enrichr (TRRUST) (http://amp.pharm.mssm.edu/Enrichr/) was used for the motif enrichment analysis (Additional file 3, Table S2). For the identification of SE, we used the bedtools toolset to identify overlaps of at least one nucleotide between H3K27ac and H3K4me1 enriched peaks. Afterwards, the ROSE algorithm [61] was used to create stitched enhancers, separating super-enhancers from typical enhancers. Finally, we used the bedtools toolset to identify overlaps of at least one nucleotide between ROSE-defined superenhancers, HDAC4 enriched peaks, and the list of SES.

\section{Statistics}

For experimental data, Student's $t$ test was employed. The Mann-Whitney test was applied when normality could not be assumed. $p<0.05$ was chosen as statistical limit of significance. For comparisons between more than two samples, the ANOVA test was applied coupled to Kruskal-Wallis and Dunn's multiple comparison test. For correlation between two variables, Pearson correlation or Spearman correlation were calculated for normal or non-normal distributions, respectively. Excel and GraphPad Prism were used for routineer analysis, R/Bioconductor packages for large data analysis and heatmap generation. We marked with ${ }^{*} p<0.05,{ }^{* * *} p<0.01$, and ${ }^{* * * *} p<0.001$. Unless otherwise indicated, all the data in the figures were represented as arithmetic means \pm the standard deviations from at least three independent experiments.

\section{Supplementary Information}

The online version contains supplementary material available at https://doi.org/10.1186/s13059-021-02340-z.

Additional file 1: Supplementary figures/All supplementary figures.

Additional file 2: Supplementary Table S1/bed formatted file of 1053 SES, identified in replicative senescence and OIS.

Additional file 3: Supplementary Table S2/List of TFs predicted to regulate the transcripts associated with SES activated and hyper-acetylated in SK-LMS-1/HDAC4 ${ }^{-/-}$cells.

Additional file 4: Supplementary Table S3/Acetylation ratio calculated between the HDAC4 KO and HDAC4 WT samples, centered on the genomic loci shown in Fig. $4 \mathrm{~g}$.

Additional file 5: Supplementary Table S4/List of the 382 SES identified by ROSE algorithm in SK-LMS-1 HDAC4 depleted cells.

Additional file 6: Supplementary Table S5/List of primers used for cloning, genome editing and PCR.

Additional file 7: Supplementary Table S6/List of the antibodies used in this paper.

Additional file 8: Supplementary Table S7/List of the genes belonging to the NFK $\beta$, SASP and RIS signatures analyzed in this manuscript.

Additional file 9. Review history. 
conditional mutant mice were generously provided by Prof. Eric N Olson and Rhonda Bassel-Duby (University of Texas Southwestern Medical Center, Dallas, Texas).

\section{Review history}

The review history is available as Additional file 9 .

\section{Authors' contributions}

CB conceived the project. EDG and CB designed and analyzed all experiments. EDG generated all knocked-out cell lines, prepared the libraries for ChIP-seq, and performed all gene expression studies. HP completed RNAi experiments and supported EDG in immunoblot experiments. EDG, ED, and RF performed the bioinformatics analysis. LR performed some western blotting. CB did the confocal microscopy. EDG, MM, HP, AR, and MM generated cell lines used in the study. EDG, ED, and CB interpreted the data. CB designed the figures with the help of EDG, ED, and RP. EDG and CB wrote the paper. The authors read and approved the final manuscript.

\section{Funding}

This study was supported by PRIN 2017 JL8SRX "Class Ila HDACs as therapeutic targets in human diseases: new roles and new selective inhibitors," Interreg Italia- Osterreich rITAT1054 EPIC and the Sarcoma Foundation of America (SFA) to $C . B$.

\section{Availability of data and materials}

Raw data corresponding to ChIP-seq experiments are uploaded with GEO accession GSE149644. For reviewers, to access the data, https://www.ncbi.nlm.nih.gov/geo/query/acc.cgi?acc=GSE149644 Enter token clyvsuiwrlafrgn into the box. Raw data corresponding to DNA microarray experiments are uploaded with GEO accession GSE150427. For reviewers, to access the data, https://www.ncbi.nlm.nih.gov/geo/query/acc.cgi?acc=GSE150427 Enter token kzybqqawhzqhfgd into the box.

\section{Declarations}

Ethics approval and consent to participate

Not applicable

\section{Consent for publication}

Not applicable

\section{Competing interests}

The authors declare no competing interests.

\section{Author details}

${ }^{1}$ Department of Medicine, Università degli Studi di Udine, p.le Kolbe 4, 33100 Udine, Italy. ${ }^{2}$ DAHFMO Unit of Histology and Medical Embryology, Sapienza University of Rome, via Antonio Scarpa 16, 00161 Rome, Italy.

Received: 29 October 2020 Accepted: 6 April 2021

Published online: 10 May 2021

\section{References}

1. Ermolaeva M, Neri F, Ori A, Rudolph KL. Cellular and epigenetic drivers of stem cell ageing. Nat Rev Mol Cell Biol. 2018; 19(9):594-610. Available from: http://www.nature.com/articles/s41580-018-0020-3. https://doi.org/10.1038/s41580-0180020-3.

2. Chan ASL, Narita M. Short-term gain, long-term pain: the senescence life cycle and cancer. Genes Dev. 2019:33(3-4):12743. Available from: http://genesdev.cshlp.org/lookup/doi/10.1101/gad.320937.118.

3. Kaneda A, Fujita T, Anai M, Yamamoto S, Nagae G, Morikawa M, et al. Activation of Bmp2-Smad1 signal and its regulation by coordinated alteration of H3K27 trimethylation in Ras-induced senescence. Barsh GS, editor. Plos Genet 2011;7:e1002359. doi: https://doi.org/10.1371/journal.pgen.1002359

4. Tasdemir N, Banito A, Roe J-S, Alonso-Curbelo D, Camiolo M, Tschaharganeh DF, et al. BRD4 connects enhancer remodeling to senescence immune surveillance. Cancer Discov. 2016;6(6):612-29. Available from: http://ca ncerdiscovery.aacrjournals.org/cgi/doi/10.1158/2159-8290.CD-16-0217.

5. Sen P, Lan Y, Li CY, Sidoli S, Donahue G, Dou Z, et al. Histone acetyltransferase p300 induces de novo super-enhancers to drive cellular senescence. Mol Cell. 2019;73:684-698.e8 Available from: https://linkinghub.elsevier.com/retrieve/pii/S1 097276519300413.

6. Paluvai H, Di Giorgio E, Brancolini C. The histone code of senescence. Cells. 2020;9:466 Available from: https://www. mdpi.com/2073-4409/9/2/466.

7. Adelman ER, Huang H-T, Roisman A, Olsson A, Colaprico A, Qin T, et al. Aging human hematopoietic stem cells manifest profound epigenetic reprogramming of enhancers that may predispose to leukemia. Cancer Discov. 2019;9(8): 1080-101. Available from: http://cancerdiscovery.aacrjournals.org/lookup/doi/10.1158/2159-8290.CD-18-1474.

8. Han R, Li L, Ugalde AP, Tal A, Manber Z, Barbera EP, et al. Functional CRISPR screen identifies AP1-associated enhancer regulating FOXF1 to modulate oncogene-induced senescence. Genome Biol. 2018;19:118 Available from: https:// genomebiology.biomedcentral.com/articles/10.1186/s13059-018-1494-1.

9. Martínez-Zamudio RI, Roux P-F, de Freitas JANLF, Robinson L, Doré G, Sun B, et al. AP-1 imprints a reversible transcriptional programme of senescent cells. Nat Cell Biol. 2020;22(7):842-55. Available from: http://www.nature.com/a rticles/s41556-020-0529-5. https://doi.org/10.1038/s41556-020-0529-5. 
10. Smith E, Shilatifard A. Enhancer biology and enhanceropathies. Nat Struct Mol Biol. 2014;21(3):210-9. Available from: http://www.nature.com/articles/nsmb.2784. https://doi.org/10.1038/nsmb.2784.

11. Alver BH, Kim KH, Lu P, Wang X, Manchester HE, Wang W, et al. The SWI/SNF chromatin remodelling complex is required for maintenance of lineage specific enhancers. Nat Commun. 2017:8:14648 Available from: http://www.nature. com/articles/ncomms14648.

12. Joshi P, Greco TM, Guise AJ, Luo Y, Yu F, Nesvizhskii Al, et al. The functional interactome landscape of the human histone deacetylase family. Mol Syst Biol. 2013;9:672 Available from: https://onlinelibrary.wiley.com/doi/abs/10.1038/ msb.2013.26

13. Xiong $Y$, Wang L, Di Giorgio E, Akimova T, Beier UH, Han R, et al. Inhibiting the coregulator CoREST impairs Foxp3+ Treg function and promotes antitumor immunity. J Clin Invest. 2020;130(4):1830-42. Available from: https://www.jci.org/a rticles/view/131375. https://doi.org/10.1172/JCl131375.

14. Stasevich TJ, Hayashi-Takanaka Y, Sato Y, Maehara K, Ohkawa Y, Sakata-Sogawa K, et al. Regulation of RNA polymerase II activation by histone acetylation in single living cells. Nature. 2014;516(7530):272-5. Available from: http://www.nature. com/articles/nature13714. https://doi.org/10.1038/nature13714.

15. Di Giorgio E, Dalla E, Franforte E, Paluvai H, Minisini M, Trevisanut M, et al. Different class lla HDACs repressive complexes regulate specific epigenetic responses related to cell survival in leiomyosarcoma cells. Nucleic Acids Res. 2020:48:646-64 Available from: https://academic.oup.com/nar/article/48/2/646/5637592.

16. Azagra A, Román-González L, Collazo O, Rodríguez-Ubreva J, de Yébenes VG, Barneda-Zahonero B, et al. In vivo conditional deletion of HDAC7 reveals its requirement to establish proper B lymphocyte identity and development. J Exp Med. 2016;213(12):2591-601. Available from: http://www.jem.org/lookup/doi/10.1084/jem.20150821.

17. Cutano V, Di Giorgio E, Minisini M, Picco R, Dalla E, Brancolini C. HDAC7-mediated control of tumour microenvironment maintains proliferative and stemness competence of human mammary epithelial cells. Mol Oncol. 2019;13:1651-68 Available from: https://onlinelibrary.wiley.com/doi/abs/10.1002/1878-0261.12503.

18. Di Giorgio E, Brancolini C. Regulation of class lla HDAC activities: it is not only matter of subcellular localization. Epigenomics. 2016;8(2):251-69. Available from: https://www.futuremedicine.com/doi/10.2217/epi.15.106.

19. Parra M. Class Ila HDACs - new insights into their functions in physiology and pathology. FEBS J. 2015;282(9):1736-44. Available from: http://doi.wiley.com/10.1111/febs.13061.

20. Marampon F, Megiorni F, Camero S, Crescioli C, McDowell HP, Sferra R, et al. HDAC4 and HDAC6 sustain DNA double strand break repair and stem-like phenotype by promoting radioresistance in glioblastoma cells. Cancer Lett. 2017;397: 1-11. Available from: https://linkinghub.elsevier.com/retrieve/pii/S0304383517302021. https://doi.org/10.1016/j.canlet.201 7.03.028.

21. Mason DX, Jackson TJ, Lin AW. Molecular signature of oncogenic ras-induced senescence. Oncogene. 2004;23:9238-46 Available from: http://www.nature.com/articles/1208172.

22. Ito T, Teo YV, Evans SA, Neretti N, Sedivy JM. Regulation of cellular senescence by polycomb chromatin modifiers through distinct DNA damage- and histone methylation-dependent pathways. Cell Rep. 2018;22(13):3480-92. Available from: https://linkinghub.elsevier.com/retrieve/pii/S2211124718303164. https://doi.org/10.1016/j.celrep.2018.03.002.

23. Chen J, Xavier S, Moskowitz-Kassai E, Chen R, Lu CY, Sanduski K, et al. Cathepsin cleavage of sirtuin 1 in endothelial progenitor cells mediates stress-induced premature senescence. Am J Pathol. 2012;180:973-83 Available from: https:// linkinghub.elsevier.com/retrieve/pii/S0002944011011023.

24. Rochelle TL, Yeung DKY, Bond MH, Li LMW. Predictors of the gender gap in life expectancy across 54 nations. Psychol Health Med. 2015;20(2):129-38. Available from: http://www.tandfonline.com/doi/abs/10.1080/13548506.2014.936884.

25. Han X, Niu J, Zhao Y, Kong Q, Tong T, Han L. HDAC4 stabilizes SIRT1 via sumoylation SIRT1 to delay cellular senescence. Clin Exp Pharmacol Physiol. 2016;43:41-6 Available from: http://www.ncbi.nlm.nih.gov/pubmed/26414199.

26. Serrano M, Lin AW, McCurrach ME, Beach D, Lowe SW. Oncogenic ras provokes premature cell senescence associated with accumulation of p53 and p16INK4a. Cell. 1997;88:593-602 Available from: https://linkinghub.elsevier.com/retrieve/ pii/S0092867400819029.

27. Kennedy AL, Morton JP, Manoharan I, Nelson DM, Jamieson NB, Pawlikowski JS, et al. Activation of the PIK3CA/AKT pathway suppresses senescence induced by an activated RAS oncogene to promote tumorigenesis. Mol Cell. 2011;42: 36-49 Available from: https://linkinghub.elsevier.com/retrieve/pii/S109727651100133X.

28. Hernandez-Segura A, Nehme J, Demaria M. Hallmarks of cellular senescence. Trends Cell Biol. 2018;28(6):436-53. Available from: https://linkinghub.elsevier.com/retrieve/pii/S0962892418300205. https://doi.org/10.1016/j.tcb.2018.02.001.

29. Deng Q, Li Y, Tedesco D, Liao R, Fuhrmann G, Sun P. The ability of E1A to rescue ras-induced premature senescence and confer transformation relies on inactivation of both p300/CBP and Rb family proteins. Cancer Res. 2005;65:8298-307 Available from: http://cancerres.aacrjournals.org/lookup/doi/10.1158/0008-5472.CAN-05-0054.

30. Cernotta N, Clocchiatti A, Florean C, Brancolini C. Ubiquitin-dependent degradation of HDAC4, a new regulator of random cell motility. Mol Biol Cell. 2011;22:278-89 Available from: http://www.molbiolcell.org/cgi/doi/10.1091/mbc. E1007-0616.

31. Parrinello S, Samper E, Krtolica A, Goldstein J, Melov S, Campisi J. Oxygen sensitivity severely limits the replicative lifespan of murine fibroblasts. Nat Cell Biol. 2003;5(8):741-7. Available from: http://www.nature.com/articles/ncb1024. https://doi.org/10.1038/ncb1024.

32. Paluvai H, Di Giorgio E, Brancolini C. Unscheduled HDAC4 repressive activity in human fibroblasts triggers TP53dependent senescence and favors cell transformation. Mol Oncol. 2018;12:2165-81 Available from: http://doi.wiley. com/10.1002/1878-0261.12392.

33. Wood KM, Roff M, Hay RT. Defective IKBa in Hodgkin cell lines with constitutively active NF-KB. Oncogene. 1998;16(16): 2131-9. Available from: http://www.nature.com/articles/1201735. https://doi.org/10.1038/sj.onc.1201735

34. Di Giorgio E, Franforte E, Cefalù S, Rossi S, Dei Tos AP, Brenca M, et al. The co-existence of transcriptional activator and transcriptional repressor MEF2 complexes influences tumor aggressiveness. McDermott JC, editor. Plos Genet. 2017;13: e1006752 Available from: https://dx.plos.org/10.1371/journal.pgen.1006752.

35. Francis AM, Alexander A, Liu Y, Vijayaraghavan S, Low KH, Yang D, et al. CDK4/6 inhibitors sensitize Rb-positive sarcoma cells to Wee1 kinase inhibition through reversible cell-cycle arrest. Mol Cancer Ther. 2017;16(9):1751-64. Available from: http://mct.aacrjournals.org/lookup/doi/10.1158/1535-7163.MCT-17-0040. 
36. Chien $Y$, Scuoppo C, Wang X, Fang X, Balgley B, Bolden JE, et al. Control of the senescence-associated secretory phenotype by NF- B promotes senescence and enhances chemosensitivity. Genes Dev. 2011;25:2125-36 Available from: http://genesdev.cshlp.org/cgi/doi/10.1101/gad.17276711.

37. Michaloglou C, Vredeveld LCW, Soengas MS, Denoyelle C, Kuilman T, van der Horst CMAM, et al. BRAFE600-associated senescence-like cell cycle arrest of human naevi. Nature. 2005;436:720-4 Available from: http://www.nature.com/articles/ nature03890.

38. Sando R, Gounko N, Pieraut S, Liao L, Yates J, Maximov A. HDAC4 governs a transcriptional program essential for synaptic plasticity and memory. Cell. 2012;151(4):821-34. Available from: https://linkinghub.elsevier.com/retrieve/pii/ S0092867412012366. https://doi.org/10.1016/j.cell.2012.09.037.

39. Di Giorgio E, Clocchiatti A, Piccinin S, Sgorbissa A, Viviani G, Peruzzo P, et al. MEF2 is a converging hub for histone deacetylase 4 and phosphatidylinositol 3-kinase/Akt-induced transformation. Mol Cell Biol. 2013;33:4473-91 Available from: http://www.pubmedcentral.nih.gov/articlerender.fcgi?artid=3838174\&tool=pmcentrez\&rendertype=abstract.

40. Inoue-Yamauchi A, Jeng PS, Kim K, Chen H-C, Han S, Ganesan YT, et al. Targeting the differential addiction to antiapoptotic BCL-2 family for cancer therapy. Nat Commun. 2017;8:16078 Available from: http://www.nature.com/articles/ ncomms 16078 .

41. Funayama R, Saito M, Tanobe H, Ishikawa F. Loss of linker histone H1 in cellular senescence. J Cell Biol. 2006;175:869-80. Available from: https://rupress.org/jcb/article/175/6/869/54751/Loss-of-linker-histone-H1-in-cellular-senescence. https:// doi.org/10.1083/jcb.200604005.

42. Hnisz D, Abraham BJ, Lee TI, Lau A, Saint-André V, Sigova AA, et al. Super-enhancers in the control of cell identity and disease. Cell. 2013;155(4):934-47. Available from: https://linkinghub.elsevier.com/retrieve/pii/S0092867413012270. https:// doi.org/10.1016/j.cell.2013.09.053.

43. Zanger K, Radovick S, Wondisford FE. CREB binding protein recruitment to the transcription complex requires growth factor-dependent phosphorylation of its GF box. Mol Cell. 2001;7(3):551-8. Available from: https://linkinghub.elsevier. com/retrieve/pii/S1097276501002027. https://doi.org/10.1016/S1097-2765(01)00202-7.

44. Lahm A, Paolini C, Pallaoro M, Nardi MC, Jones P, Neddermann P, et al. Unraveling the hidden catalytic activity of vertebrate class Ila histone deacetylases. Proc Natl Acad Sci. 2007;104(44):17335-40. Available from: http://www.pnas. org/cgi/doi/10.1073/pnas.0706487104.

45. Seto E, Yoshida M. Erasers of histone acetylation: the histone deacetylase enzymes. Cold Spring Harb Perspect Biol. 2014;6(4):a018713. Available from: http://cshperspectives.csh/p.org/lookup/doi/10.1101/cshperspect.a018713.

46. Eferl R, Wagner EF. AP-1: a double-edged sword in tumorigenesis. Nat Rev Cancer. 2003;3:859-68 Available from: http:// www.nature.com/articles/nrc1209.

47. Parry AJ, Hoare M, Bihary D, Hänsel-Hertsch R, Smith S, Tomimatsu K, et al. NOTCH-mediated non-cell autonomous regulation of chromatin structure during senescence. Nat Commun. 2018;9:1840 Available from: http://www.nature. com/articles/s41467-018-04283-9.

48. Muñoz-Espín D, Cañamero M, Maraver A, Gómez-López G, Contreras J, Murillo-Cuesta S, et al. Programmed cell senescence during mammalian embryonic development. Cell. 2013;155:1104-18 Available from: http://www.ncbi.nlm. nih.gov/pubmed/24238962

49. Marzagalli M, Casati L, Moretti RM, Montagnani Marelli M, Limonta P. Estrogen receptor $\beta$ agonists differentially affect the growth of human melanoma cell lines. Weisz A, editor. Plos One 2015;10:e0134396. Available from: http://dx.plos. org/10.1371/journal.pone.0134396

50. Potthoff MJ, Wu H, Arnold MA, Shelton JM, Backs J, McAnally J, et al. Histone deacetylase degradation andMEF2 activation promote the formation of slow-twitch myofibers. J Clin Invest. 2007;117(9):2459-67. Available from: http:// www.jci.org/cgi/doi/10.1172/JCl31960

51. Nam Y-J, Lubczyk C, Bhakta M, Zang T, Fernandez-Perez A, McAnally J, et al. Induction of diverse cardiac cell types by reprogramming fibroblasts with cardiac transcription factors. Development. 2014;141(22):4267-78. Available from: http:// dev.biologists.org/cgi/doi/10.1242/dev.114025.

52. Di Giorgio E, Gagliostro E, Clocchiatti A, Brancolini C. The control operated by the cell cycle machinery on MEF2 stability contributes to the downregulation of CDKN1A and entry into S phase. Mol Cell Biol. 2015;35:1633-47 Available from: http://mcb.asm.org/lookup/doi/10.1128/MCB.01461-14.

53. Clocchiatti A, Di Giorgio E, Viviani G, Streuli C, Sgorbissa A, Picco R, et al. The MEF2-HDAC axis controls proliferation of mammary epithelial cells and acini formation in vitro. J Cell Sci. 2015;128(21):3961-76. Available from: http://jcs. biologists.org/cgi/doi/10.1242/jcs.170357.

54. Peruzzo P, Comelli M, Di Giorgio E, Franforte E, Mavelli I, Brancolini C. Transformation by different oncogenes relies on specific metabolic adaptations. Cell Cycle. 2016;15(19):2656-68. Available from: https://www.tandfonline.com/doi/full/1 $0.1080 / 15384101.2016 .1215387$.

55. Wienert B, Shin J, Zelin E, Pestal K, Corn JE. In vitro-transcribed guide RNAs trigger an innate immune response via the RIG-I pathway. Bhandoola A, editor. Plos Biol 2018;16:e2005840. Available from: https:/dx.plos.org/10.1371/journal.pbio.2005840

56. Paroni G, Mizzau M, Henderson C, Del Sal G, Schneider C, Brancolini C. Caspase-dependent regulation of histone deacetylase 4 nuclear-cytoplasmic shuttling promotes apoptosis. Mol Biol Cell. 2004;15:2804-18 Available from: http:// www.ncbi.nlm.nih.gov/pubmed/15075374.

57. Lasry A, Ben-Neriah Y. Senescence-associated inflammatory responses: aging and cancer perspectives. Trends Immunol. 2015;36:217-28 Available from: https://linkinghub.elsevier.com/retrieve/pii/S147149061500040X.

58. Cerami E, Gao J, Dogrusoz U, Gross BE, Sumer SO, Aksoy BA, et al. The cBio Cancer Genomics Portal: an open platform for exploring multidimensional cancer genomics data: figure 1. Cancer Discov. 2012;2(5):401-4. Available from: http://ca ncerdiscovery.aacrjournals.org/lookup/doi/10.1158/2159-8290.CD-12-0095.

59. Shao Z, Zhang Y, Yuan G-C, Orkin SH, Waxman DJ. MAnorm: a robust model for quantitative comparison of ChIP-Seq data sets. Genome Biol. 2012;13:R16 Available from: http://genomebiology.biomedcentral.com/articles/10.1186/gb-2 012-13-3-r16.

60. Jiang Y, Qian F, Bai X, Liu Y, Wang Q, Ai B, et al. SEdb: a comprehensive human super-enhancer database. Nucleic Acids Res. 2019;47:D235-43. Available from: https://academic.oup.com/nar/article/47/D1/D235/5146197. https://doi.org/10.1 093/nar/gky1025 
61. Whyte WA, Orlando DA, Hnisz D, Abraham BJ, Lin CY, Kagey MH, et al. Master transcription factors and mediator establish super-enhancers at key cell identity genes. Cell. 2013;153(2):307-19. Available from: https://inkinghub.elsevier. com/retrieve/pii/S0092867413003929. https://doi.org/10.1016/j.cell.2013.03.035.

62. Quinlan AR, Hall IM. BEDTools: a flexible suite of utilities for comparing genomic features. Bioinformatics. 2010;26(6):8412. Available from: https://academic.oup.com/bioinformatics/article-lookup/doi/10.1093/bioinformatics/btq033.

63. Bailey TL, Johnson J, Grant CE, Noble WS. The MEME suite. Nucleic Acids Res. 2015;43(W1):W39-49. Available from: https://academic.oup.com/nar/article-lookup/doi/10.1093/nar/gkv416.

\section{Publisher's Note}

Springer Nature remains neutral with regard to jurisdictional claims in published maps and institutional affiliations.

Ready to submit your research? Choose BMC and benefit from:

- fast, convenient online submission

- thorough peer review by experienced researchers in your field

- rapid publication on acceptance

- support for research data, including large and complex data types

- gold Open Access which fosters wider collaboration and increased citations

- maximum visibility for your research: over $100 \mathrm{M}$ website views per year

At $\mathrm{BMC}$, research is always in progress.

Learn more biomedcentral.com/submissions 